\title{
Molecular Mechanism of Hippo-YAP1/TAZ Pathway in Heart Development, Disease, and Regeneration
}

\author{
Xiaoqing Chen ${ }^{1,2}$, Yilang $\mathrm{Li}^{1,2}$, Jiandong Luo ${ }^{1,2 *}$ and Ning Hou ${ }^{1,2 *}$ \\ ${ }^{1}$ Key Laboratory of Molecular Target \& Clinical Pharmacology, School of Pharmaceutical Sciences and the Fifth Affiliated \\ Hospital, Guangzhou Medical University, Guangzhou, China, ${ }^{2}$ Guangzhou Institute of Cardiovascular Disease, Guangzhou \\ Key Laboratory of Cardiovascular Disease, and The Second Affiliated Hospital of Guangzhou Medical University, Guangzhou, \\ China
}

\section{OPEN ACCESS}

Edited by:

Mark J. Ranek,

Johns Hopkins University,

United States

Reviewed by:

Hyun Woo Park,

Yonsei University, South Korea

Dominic Del Re,

Rutgers, The State University

of New Jersey, United States

${ }^{*}$ Correspondence:

Ning Hou

houning@gzhmu.edu.cn

Jiandong LuO

jiandongluo@hotmail.com

Specialty section:

This article was submitted to

Striated Muscle Physiology,

a section of the journal

Frontiers in Physiology

Received: 15 November 2019

Accepted: 01 April 2020

Published: 23 April 2020

Citation:

Chen X, Li Y, Luo J and Hou N

(2020) Molecular Mechanism

of Hippo-YAP1/TAZ Pathway in Heart

Development, Disease,

and Regeneration.

Front. Physiol. 11:389.

doi: 10.3389/fphys.2020.00389
The Hippo-YAP1/TAZ pathway is a highly conserved central mechanism that controls organ size through the regulation of cell proliferation and other physical attributes of cells. The transcriptional factors Yes-associated protein 1 (YAP1) and PDZ-binding motif (TAZ) act as downstream effectors of the Hippo pathway, and their subcellular location and transcriptional activities are affected by multiple post-translational modifications (PTMs). Studies have conclusively demonstrated a pivotal role of the Hippo-YAP1/TAZ pathway in cardiac development, disease, and regeneration. Targeted therapeutics for the YAP1/TAZ could be an effective treatment option for cardiac regeneration and disease. This review article provides an overview of the Hippo-YAP1/TAZ pathway and the increasing impact of PTMs in fine-tuning YAP1/TAZ activation; in addition, we discuss the potential contributions of the Hippo-YAP1/TAZ pathway in cardiac development, disease, and regeneration.

Keywords: YAP1/TAZ, Hippo pathway, post-translational modification, heart development, cardiac disease, regeneration

\section{INTRODUCTION}

The Hippo pathway, originally identified in the Drosophila genus, is a highly conserved kinase cascade that regulates organ size (Harvey et al., 2003; Pan, 2007; Hayashi et al., 2015). The transcriptional coactivator Yes-associated protein 1 (YAP1; homolog of Yorkie) was first identified as a binding partner of the SH3 domain of c-yes; the YAP1 and its paralog PDZ-binding motif (TAZ; also known as WW-domain-containing transcription regulator 1 [WWTR1]), are both downstream effectors of Hippo signaling (Sudol, 1994). This pathway has been implicated in diverse biological functions, both in Drosophila and in mammals; these include cell proliferation, apoptosis, organ-size control, and cancer progression (Mo, 2017). Recent reports have revealed the critical role of YAP1/TAZ in cardiac development, regeneration, and stress response; however, there are some inconsistent and even contradictory results that warrant further investigation (Zhou et al., 2015). Interestingly, recent studies have identified a variety of post-translational modifications (PTMs) 
to YAP1/TAZ, such as phosphorylation, O-GlcNacylation, methylation, and ubiquitination, which offers an opportunity to control the Hippo-YAP1/TAZ pathway. In this review, we summarize the overall picture of the Hippo-YAP1/TAZ pathway; in particular, we highlight the novel discoveries with regard to PTM-related regulation and the function of the YAP1/TAZ pathway in cardiac development, disease and regeneration.

\section{OVERVIEW OF THE HIPPO-YAP1/TAZ PATHWAY}

\section{The Canonical Hippo-YAP1/TAZ Pathway in Mammals}

The components of the Hippo pathway in mammals are highly consistent with those in Drosophila, including sterile 20-like protein kinases (MST1/2; homologs of D. Hpo), salvador family protein 1 (SAV1; which contains a WW domain), large tumor suppressors (LATS1/2; homologs of Wts), Mps one binder kinase activator-like 1A/1B (MOB1; orthologs of Mats), YAP1 /TAZ, and other peripheral proteins (Figure 1).

The upstream signals and stressors of the Hippo pathway include cell polarity, energy stresses, G-protein-coupled receptors (GPCRs), and stiffness of the extracellular matrix (ECM) (Meng et al., 2016). On stimulation, MST1/2 and its adaptor protein SAV1 are phosphorylated leading to their activation; subsequently, these stimulate the phosphorylation, and activation of the LATS1/2-MOB1 complex. Through an interaction between the PPxY (PY) motifs of LATS1/2 and the WW domains of YAP1/TAZ, the activated LATS1/2 can induce phosphorylation of YAP1 (mouse YAP1 at Ser112, human YAP1 at Ser127) and TAZ (Ser89) (Zhao et al., 2007). After phosphorylation, YAP1/TAZ binds to the 14-3-3 protein, which induces cytoplasmic retention of YAP1/TAZ. Moreover, phosphorylated YAP1/TAZ is likely to undergo ubiquitination degradation that is dependent on the $\beta$-transducin repeat-containing E3 ubiquitin protein ligase complex (SCF ${ }^{\beta-\mathrm{TRCP}}$ ) (Dong et al., 2007; Zhao et al., 2007).

When Hippo signaling is inactive, the YAP1/TAZ are activated and mainly localized in the nucleus, where they produce downstream biological effects. The YAP1/TAZ do not harbor DNA-binding domains; instead, these interact with transcriptional partner proteins to initiate or impede transcription. The transcriptional partners of YAP1/TAZ that have been identified thus far include transcriptional enhanced associate domain proteins (TEADs), members of the Smad family (Anorga et al., 2018; Ben Mimoun and Mauviel, 2018; Miskolczi et al., 2018; Qin et al., 2018), and p63/p73 (Levy et al., 2008; Kim et al., 2018). The onus of the YAP1/TAZ biological output rests with the transcriptional partners and the target genes that they modulate.

\section{Downstream Output of YAP1/TAZ}

As orthologs of Drosophila Scalloped, the TEAD family of proteins in mammals comprise YAP1/TAZ- and DNA-binding domains, which facilitates the construction of a YAP1/TAZTEADs co-transcription complex (Li et al., 2010). Recent studies investigating YAP1/TAZ mechanisms have identified some target genes of the YAP1/TAZ-TEADs complex. The glucose transporter 1 (Glut1) promotor contains TEAD-binding motifs that allow Glut1 to be regulated by the YAP1-TEAD1 transcriptional complex, which enhances cell glycolysis in breast cancer cells (Valis et al., 2016; Lin and Xu, 2017). Moreover, GLUT1, a transmembranous protein, participates in cell glucose uptake (Wang et al., 2017). Left ventricular hypertrophy is characterized by enhanced GLUT1 expression and basal glucose uptake in heart tissues. Cardiac-specific overexpression of GLUT1 can retard the progression of heart failure and reduce mortality associated with pressure overload (PO) (Liao et al., 2002). Thus, YAP1/TAZ may have a cardioprotective effect by enhancing GLUT1 expression. In breast cancer cells, the G2 and $S$ phaseexpressed 1 (GTSE1) acts as a novel target gene of the YAP1TEAD4 complex, which forms cell protrusions and boosts cell migration (Stelitano et al., 2017). The GTSE1 is a microtubuleassociated protein that is involved in cell proliferation, invasion, and migration (Liu et al., 2019). Therefore, through regulation of GTSE1, YAP1 facilitates epithelial-to-mesenchymal transition (EMT) in cancer cells.

Furthermore, the YAP1/TAZ-TEAD complex can recruit and bind to transcription enhancers and/or mediators to potentiate and blunt transcription. Prox1, a major regulator of lymphangiogenesis, is regulated by the YAP1/TAZ-TEAD complex (Harvey et al., 2005; Cho et al., 2019). The YAP1/TAZTEAD transcriptional complex recruits the nucleosome remodeling and histone deacetylase (NuRD) complex to negatively modulate Prox 1 ; this was shown to reduce pathological lymphangiogenesis and maintain the lymphatic valve postnatally (Cho et al., 2019). In addition, the YAP1/TAZ, together with histone deacetylase 7 (HDAC7) and TEAD, binds to the COX-2 promoter and attenuates $C O X-2$ transcription in order to inhibit IL-1 $\beta$-induced cell migration and invasion (Zhang et al., 2018a). Based on its tyrosine phosphorylation status, parafibromin (a nuclear scaffold protein) selectively interacts with YAP1 or TAZ. Phosphorylated parafibromin binds to the YAP1-TEAD complex, whereas dephosphorylated parafibromin combines with the TAZ-TEAD transcriptional activator (Tang et al., 2018). YAP1 and TAZ have largely overlapping transcriptional functions, such as cell proliferation and cell migration; however, both have unique biological functions (Lai et al., 2018; Liu et al., 2018; Negron-Perez and Hansen, 2018). For example, YAP1 knockout (KO), rather than TAZ KO, in mice leads to embryonic death (Hossain et al., 2007; Makita et al., 2008). Conversely, parafibromin regulates the activity of YAP1 and TAZ in different status, which may explain the unique functions of YAP1 and TAZ.

Newly discovered co-transcription proteins reportedly contribute to YAP1/TAZ functions. Pyruvate kinase M2 (PKM2) is a relevant glycolytic protein; the interaction of YAP1 with hypoxia-inducible factor $1 \alpha$ (HIF-1 $\alpha$ ) promotes tumor cell glycolysis by triggering the transcription of the Pkm2 gene (Zhang et al., 2018b). YAP1-mediated PKM2 expression enhances cell glycolysis and adapts tumor cells for 


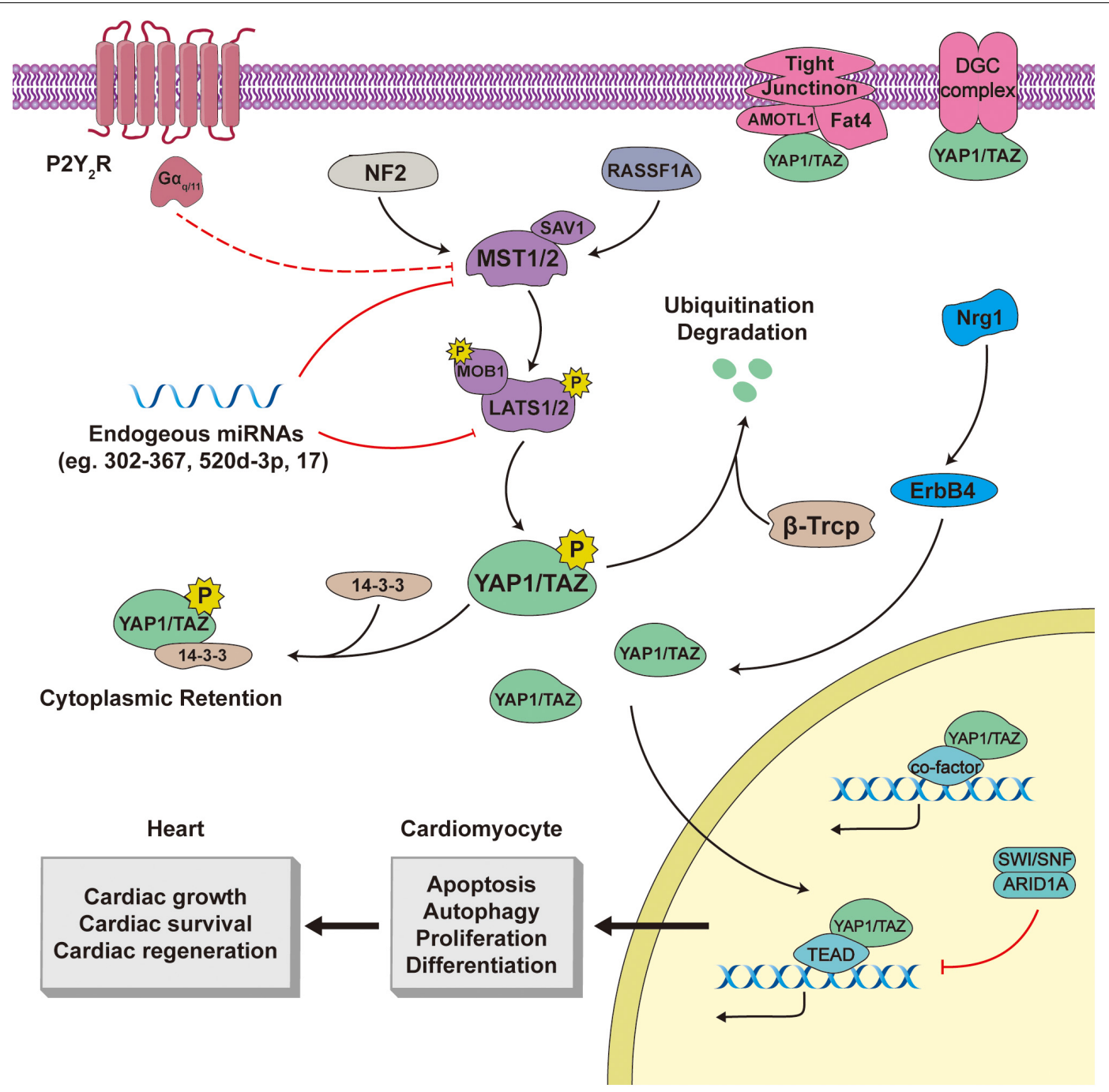

FIGURE 1 | The Hippo-YAP1/TAZ pathway in cardiac biology. Both YAP1 and TAZ are phosphorylated by core components, including Mst1/2, SAV1, Lats1/2, and MOB1, of the canonical Hippo pathway, and are subsequently degraded or stranded in the cytoplasm. Active YAP1/TAZ translocate to the nucleus, bind to transcriptional partners, and modulate downstream output in cardiac tissue. In addition, the Hippo-YAP1/TAZ pathway is regulated by several upstream pathways and cell-cell junctions. Line arrows indicate activation, whereas connector lines imply inhibition.

abnormal growth. The SWI/SNF (Brg/Brahma-associated factors [BAF]) and ARID1A (BAF250A) proteins are inhibitors of the YAP1/TAZ-TEADs complex; in addition, these are capable of occupying the TEAD-binding site of YAP1/TAZ (Chang et al., 2018). Although the ARID1A-SWI/SNF complex is inactive during tumor growth, this negative association highlights the role of the ARID1A-SWI/SNF complex in cancer development through the suppression of oncogenic YAP1/TAZ activity (Kadoch and Crabtree, 2015).

Collectively, novel transcription mechanisms have been reported for explaining partly YAP1/TAZ biological output; these findings bode well for future YAP1/TAZ studies. However, the diversity of transcription partners and the target genes of YAP1/TAZ make it difficult to clarify the biological effects of YAP1/TAZ; therefore, future research should consider the entire molecular network of the YAP1/TAZ pathway.

\section{REGULATION OF THE YAP1/TAZ BY POST-TRANSLATIONAL MODIFICATIONS}

\section{Regulation of the YAP1/TAZ by Phosphorylation}

Yes-associated protein 1 and TAZ, two salient downstream effectors of Hippo signaling, are found both in the cytoplasm and nucleus. Since these two effectors trigger target gene transcription in the nucleus, nuclear accumulation of YAP1/TAZ is a key step in their mechanism of action. This nuclear accumulation is mediated by several events: (1) posttranslational modifications (PTMs) including phosphorylation, methylation, and ubiquitination; (2) other related protein interactions with YAP1/TAZ; and (3) translational interference 
(Piccolo et al., 2014; Mo, 2017). In most scenarios, YAP1/TAZ are mediated by PTMs that determine their subcellular localization and content (Table $\mathbf{1}$ ).

In the canonical Hippo pathway, phosphorylation plays a pivotal role in the regulation of YAP1/TAZ; they can be phosphorylated at multiple sites, such as Ser61, Ser109, Ser127 (Ser89 in TAZ), Ser164, and Ser381 (Ser311 in TAZ) (Zhao et al., 2010). Among these, Ser127 and Ser381 are the main phosphorylation sites by LATS1/2. Following phosphorylation at Ser381, YAP1 is prone to further phosphorylation by the casein kinase I isoform $\delta / \varepsilon(\mathrm{CKI} \delta / \varepsilon)$, which eventually promotes $\mathrm{SCF}^{\beta-\mathrm{TRCP}}$-induced ubiquitination degradation of YAP1 (Zhao et al., 2010). YAP1 binds to 14-3-3 protein through the Ser127 phosphorylation-dependent way and becomes sequestered in the cytoplasm (Zhao et al., 2007). Notably, the combination of YAP1 and 14-3-3 can be blocked by phosphorylation at the YAP1 Ser128 site by Nemo-like kinase (NLK); this phenomenon can also be seen in Drosophila Nemo (Moon et al., 2017). This indicates that NLK/Nemo are highly conserved in HippoYAP1/TAZ signaling to mediate the YAP1/Yki subcellular compartmentation via phosphorylation.

Phosphorylation-dependent cytoplasmic retention of YAP1/TAZ may hamper their transcriptional activity. In the transgenic heart, MST1 overexpression was shown to enhance the phosphorylation of YAP1 Ser127 and induce inactivation of YAP1; this induced cardiomyocyte (CM) apoptosis and myocardial fibrosis (Yamamoto et al., 2003; Zhao et al., 2019). In LATS1/2 cardiac knock out (CKO) of the embryonic epicardium, LAST1/2 deficiency was shown to inhibit the phosphorylation of YAP1 Ser127 and increase the YAP1 transcriptional activity; eventually, this resulted in the failure of subepicardial cells to differentiate into cardiac fibroblasts and induced coronary vessel defects (Xiao et al., 2018). Thus, the canonical Hippo pathway inhibits the YAP1/TAZ activity in cardiac tissues through phosphorylation. Interestingly, YAP1/TAZ was shown to increase the concentration and activity of endogenous LATS2 by directly inducing TEAD-dependent LATS2 transcription and/or indirectly activating neurofibromin 2 (NF2)-mediated LATS2 activity, which subsequently enhances the phosphorylation of YAP1/TAZ (Moroishi et al., 2015; Dai et al., 2017). This physiological negative feedback mechanism is expected to maintain the dynamic homeostasis of the Hippo pathway, and may help elucidate the complex mechanism of cardiac development and disease.

Notably, YAP1/TAZ can be dephosphorylated by a catalytic subunit of protein phosphatase-1 (PP1A) at Ser127 (Ser89 and Ser311 in TAZ), and is then translocated into the nucleus, thereby causing leak activation of the target gene (Liu et al., 2011; Wang et al., 2011). This abnormal activation can be potentially prevented by some mechanisms. The pre-mRNA splicing factor 4 kinase (PRP4K) promotes phosphorylation of Yki/YAP1/TAZ in the nucleus as well as its subsequent nuclear export; this acts as a safety threshold for the rescue of aberrant Yki/YAP1/TAZ activities and cell growth (Cho et al., 2018).

Besides serine phosphorylation, the subcellular distribution and activities of YAP1/TAZ are affected by the phosphorylation of tyrosine residues. Several members of the SRC family of kinases (SFK), such as Yes, Src, and c-Abl, participate in

TABLE 1 | Post-translational modifications that modulate YAP1/TAZ.

\begin{tabular}{|c|c|c|c|}
\hline Modification types & Regulative sites & Enzymes & Effects \\
\hline \multirow[t]{8}{*}{ Phosphorylation } & Ser109 & LATS1/2 & YAP1/TAZ cytoplasm retention (Zhao et al., 2010) \\
\hline & Ser127 (Ser89 in TAZ) & LATS1/2 & Binding YAP1 to 14-3-3 and inducing cytoplasm retention (Zhao et al., 2007) \\
\hline & Ser128 & NLK & Blocking YAP1 binding to 14-3-3 (Moon et al., 2017) \\
\hline & Ser381 (Ser311 in TAZ) & LATS1/2 & $\beta$-Trcp-dependent ubiquitination degradation (Zhao et al., 2010) \\
\hline & NS & PRP4K & YAP1/TAZ nuclear export (Cho et al., 2018) \\
\hline & Tyr357 & c-Abl & $\begin{array}{l}\text { Stabilizing YAP1 stable and increasing YAP1-p73 interaction inducing cell apoptosis } \\
\text { (Levy et al., 2008) }\end{array}$ \\
\hline & Tyr357 & Src & Enhancing YAP1 nuclear retention and target gene CTGF expression (Taniguchi et al., 2015) \\
\hline & one or more tyrosine residues & Yes & Promoting YAP1 translocation and Oct-3/4 transcription (Tamm et al., 2011) \\
\hline Dephosphorylation & Ser127 (Ser89 and Ser311 in TAZ) & PP1A & YAP1/TAZ nuclear translocation (Liu et al., 2011; Wang et al., 2011). \\
\hline \multirow[t]{2}{*}{ O-GlcNacylation } & Ser109 & OGT & $\begin{array}{l}\text { Impeding LATS1/2-induced YAP1 Ser109 phosphorylation and potentiating YAP1 activity } \\
\text { (Peng et al., 2017) }\end{array}$ \\
\hline & Thr241 & OGT & $\begin{array}{l}\text { Attenuating LATS1/2-dependent YAP1 Ser127 phosphorylation and enhancing YAP1 } \\
\text { activity (Zhang et al., 2017) }\end{array}$ \\
\hline \multirow[t]{2}{*}{ Methylation } & Lys342 & SET1A & Dampening YAP1-CRM1 fusion and enhancing YAP1 nuclear retention (Fang et al., 2018) \\
\hline & Lys494 & SET7 & Enhancing YAP1 translocation to cytosol and cytoplasmic membrane Oudhoff et al., 2013) \\
\hline \multirow[t]{3}{*}{ Ubiquitination } & NS & SCF ${ }^{\beta-T R C P}$ & YAP1/TAZ protein degradation (Dong et al., 2007; Zhao et al., 2007) \\
\hline & NS & Fbxw7 & YAP1 protein degradation (Tu et al., 2014; Zhang et al., 2016) \\
\hline & Lys321, Lys497 & SKP2 & $\begin{array}{l}\text { Accumulating YAP1 nuclear localization and association between YAP1 and TEAD } \\
\text { (Yao et al., 2018) }\end{array}$ \\
\hline \multirow[t]{2}{*}{ De-ubiquitination } & NS & USP9X & Stabilizing YAP1 and enhancing its activity (Li et al., 2018a). \\
\hline & NS & OTUD1 & $\begin{array}{l}\text { Reducing YAP1 stability and attenuating the cell proliferation activity of YAP1 } \\
\text { (Yao et al., 2018). }\end{array}$ \\
\hline
\end{tabular}

NS, not specified, where the specific regulator sites have not been validated. 
tyrosine phosphorylation of YAP1. In response to DNA damage, YAP1 Tyr357 phosphorylation by c-Abl stabilizes YAP1 and increases the nuclear interaction of YAP1-p73 to induce apoptosis (Levy et al., 2008). This phosphorylation may inhibit the cell proliferation and oncogenic ability of YAP1 that are necessary to sustain its normal biological functions. The activated Yes phosphorylates YAP1 on one or more tyrosine residues; the phosphorylated YAP1 is subsequently translocated to the nucleus to trigger the transcription of the pluripotency factor Oct$3 / 4$, which is critical for maintaining the self-renewal ability of the mouse and human embryonic stem cells (hESCs) (Tamm et al., 2011). Furthermore, Src phosphorylated by the IL6-GP130 complex phosphorylates YAP1 at Tyr357, which increases YAP1 nuclear retention and expression of its target gene connective tissue growth factor (CTGF); this Src-induced YAP phosphorylation facilitates the regeneration of the injured intestinal mucosa (Taniguchi et al., 2015). The above-mentioned research shows that tyrosine phosphorylation participates in the regulation of YAP1/TAZ in specific cell and tissue regeneration. This suggests that the tyrosine phosphorylation of YAP1/TAZ may be conductive to embryonic cardiac development and postinjury cardiac regeneration; these findings provide new insights into cardiac development and regeneration.

\section{Regulation of the YAP1/TAZ by O-GIcNAcylation}

Besides phosphorylation, several PTMs, such as $\mathrm{O}-$ GlcNAcylation, are involved in the regulation of YAP1/TAZ. O-GlcNAc transferase (OGT) directly induces O-GlcNAcylation at YAP1 Ser109 and Thr241, and potentiates the pro-proliferation activity of YAP1 in pancreatic cancer and liver cancer cells (Peng et al., 2017; Zhang et al., 2017). Mechanistically, YAP1 O-GlcNAcylation can affect YAP1 phosphorylation. As mentioned in Section "Regulation of the YAP1/TAZ by Phosphorylation," LATS1/2 can phosphorylate YAP1 Ser109 (Zhao et al., 2010). Therefore, O-GlcNAcylation and phosphorylation may act competitively at Ser109. The stoichiometry of Ser109 phosphorylation is much lower than that of O-GlcNAcylation, which suggests that O-GlcNAcylation plays a key role in PTM at this site rather than phosphorylation (Peng et al., 2017). O-GlcNAcylation at YAP1 Thr241 can attenuate the phosphorylation of YAP1 at Ser127 via interrupting the LATS1/2 interaction (Zhang et al., 2017). In addition, these studies reveal that YAP1 not only acts as a substrate of OGT but also triggers OGT transcription to regulate its expression, thereby forming a positive feedback loop (Peng et al., 2017; Zhang et al., 2017). This positive feedback loop further regulates YAP1 activity and output.

Therefore, YAP1 may participate in cancer glycosylation metabolism through O-GlcNAcylation and OGT expression in order to maintain the abnormal proliferation and survival of cancer cells. In vitro, OGT KO inhibits O-GlcNAcylation at the YAP1 Ser109, thereby inhibiting cell-colony formation in pancreatic cancer; in vivo, OGT knockdown downregulates tumor growth (Peng et al., 2017). High glucose stimulation improves the level of O-GlcNAcylation at YAP1 Thr241; in addition, O-GlcNAcylation enhances the stability and activity of YAP1, which subsequently induces cell proliferation and transformative phenotypes in hepatocellular carcinoma cells (HCC, THLE-3, and HL-7702 cell lines) (Zhang et al., 2017). In addition to liver cancer, high glucose level affects diabetic cardiomyopathy. Elevated cardiac concentrations of active YAP1 have been demonstrated in mice fed with highfat diet and in patients with diabetic cardiomyopathy (Ikeda et al., 2019b). However, the relationship between YAP1 and diabetic cardiomyopathy is not completely clear. Thus, YAP1 O-GlcNAcylation might be a promising target for the study of diabetic cardiomyopathy.

\section{Regulation of the YAP1/TAZ by Ubiquitination}

The stability and content of YAP1/TAZ are partly regulated by ubiquitination-induced degradation; therefore, ubiquitination cannot be neglected in research into YAP1/TAZ. SCF ${ }^{\beta-T R C P}$ dependent ubiquitination is a well-known YAP1/TAZ degradation mechanism in the canonical Hippo pathway that is dependent on YAP1/TAZ phosphorylation (Dong et al., 2007; Zhao et al., 2007). Similarly, Fbxw7, another subunit of E3 ligase, participates in the regulation of YAP1 protein level and activity via ubiquitination and proteasomal degradation (Tu et al., 2014; Zhang et al., 2016). Fbxw7 expression exhibits a negative correlation with YAP1 content in human HCC and pancreatic ductal adenocarcinoma tissues; however, the regulatory mechanisms are yet to be elucidated. This proteolytic ubiquitination can be reversed by deubiquitinases; the deubiquitinase USP9X was shown to directly deubiquitinate and stabilize YAP1, leading to enhanced YAP1 activity and promotion of tumor growth (Li et al., 2018a). LATS1/2, activated by mono-ubiquitinated angiomotin-like 2 (AMOTL2), triggers YAP1 phosphorylation and suppresses its activity (Kim et al., 2016). USP9X deubiquitinates AMOTL2 to inactivate LATS1/2, which indirectly activates YAP1 (Kim et al., 2016). Therefore, ubiquitination appears to be a negative regulatory factor for YAP1/TAZ in the canonical Hippo pathway.

Intriguingly, recent studies have provided new insights into ubiquitination independent of the Hippo pathway. The $\mathrm{SCF}^{\mathrm{SKP} 2} \mathrm{E} 3$ ligase complex (SKP2) facilitates K63-linkagespecific ubiquitination of YAP1 at Lys321 and Lys497, which does not reduce YAP1 abundance (Yao et al., 2018). Moreover, these ubiquitination promotes nuclear translocation of YAP1, and cements the association between YAP1 and TEAD to enhance the cell proliferation activity of YAP1 (Yao et al., 2018). This non-proteolytic ubiquitination can be reversed by the binding and interaction between deubiquitinase OTUD1 and YAP1; OTUD1 decreases YAP1 stability, and attenuates the cell proliferation and tumor-growth activity of YAP1 (Yao et al., 2018). Obviously, this non-proteolytic ubiquitination is beneficial for YAP1 activation and output. The above-mentioned studies delineate the controversial role of ubiquitination in YAP1/TAZ in the canonical Hippo-YAP1/TAZ pathway as well as in the Hippo-independent pathway. YAP1/TAZ is degraded by 
ubiquitinases that exert proteolytic effects, whereas it is stabilized by ubiquitinases with non-proteolytic activities; these events can be reversed by the related deubiquitinases. Undoubtedly, ubiquitination plays an essential role in mediating YAP1/TAZ content and stability that should be further studied. The biological effects of ubiquitinated/deubiquitinated YAP1/TAZ have been investigated mainly in tumorigenesis, and less so in cardiogenesis and cardiac disease.

\section{Regulation of the YAP1/TAZ by Methylation}

Chromosomal maintenance 1 (CRM1), a nuclear export protein, triggers the nuclear export of YAP1. The methyltransferase SET1A targets YAP1 at Lys342 through mono-methylation, which inhibits CRM1-induced nuclear export of YAP1 with resultant enhancement of YAP1 nuclear retention and transcriptional activity (Fang et al., 2018). Mono-methylation at Lys342 in YAP1 contributes to in vivo cell proliferation and tumorigenesis (Fang et al., 2018). However, this result was discordant with that from a previous study on mono-methylated YAP1. The SET7 is a methyltransferase that connects the cell membrane and the cytoskeleton (Garbino et al., 2009). Mono-methylation of YAP1 Lys494 by SET7 is necessary for the retention of YAP1 in the cytosol and at the cytoplasmic membrane, which may impede interactions between YAP1 and PDZ-dependent-binding partners (Oudhoff et al., 2013). Thus, methylation at YAP1 Lys494 represses YAP1 activity to downregulate CTGF transcription. Evidently, methylation can exert certain effects on YAP1/TAZ, but there is a paucity of studies on YAP1/TAZ methylation and their biological output. In addition, the mechanisms by which methylated YAP1/TAZ affect cardiac biology remain unknown.

Several types of PTMs alter the subcellular translocation of YAP1/TAZ to regulate their activities and output, regardless of how YAP1/TAZ are modified by PTMs. Therefore, it is important to study the nuclear-cytoplasmic trafficking of YAP1/TAZ. An increasing body of evidence supports the mechanisms of YAP1/TAZ nucleocytoplasmic shuttling. In a stiff cultured environment, the nucleus encounters mechanical forces and becomes flattened; in addition, the nuclear pores are stretched, subsequently creating conditions for nuclear import of YAP1 (Elosegui-Artola et al., 2017). A nuclear localization sequence (NLS) and nuclear export sequence (NES) were identified within TAZ, which contributes to the nucleocytoplasmic distribution of TAZ in different ways: TEAD binding interaction partly conceals the NES; RhoA enhances NLS-induced nuclear import; and TEAD and 14-3-3 competitively bind with TAZ (Kofler et al., 2018).

Collectively, PTMs can alter YAP1/TAZ stability and/or YAP1/TAZ conformation to change its affinity for other proteins; these eventually mediate YAP1/TAZ subcellular location and content. PTMs mediate YAP1/TAZ through interdependent, synergistic, or competitive mechanisms. A growing body of evidence has revealed the underlying mechanisms of PTMs and YAP1/TAZ activity; however, several important nuances are yet to be elucidated.

\section{HIPPO-YAP1/TAZ SIGNALING IN CARDIAC DEVELOPMENT}

Cardiogenesis involves events that alter the spatiotemporal and morphological mechanisms. In the gastrulation period, the mesoderm, which emanates from the anterior primitive streak (APS), produces cardiac progenitor cells (Tam et al., 1997). Subsequently, cardiac progenitor cells form two different heart fields: the primary heart field gives rise to the left ventricle and left/right atria, while the secondary heart field generates the right ventricle, left/right atria, and the outflow tract (Xin et al., 2013b). Postnatally, there is a decrease in CM proliferation alongside an increase in hypertrophy which helps attain the physiologic cardiac size. At approximately postnatal Day 4 (P4), myocardial cell numbers peak, and then stop increasing (Li et al., 1996). Myocardial proliferation can be blunted as early as E11.5 with the help of cardiac gene reprogram; this results in abnormal cardiac growth and perinatal lethality (Wang et al., 2014).

\section{Role of Hippo-YAP1/TAZ Signaling and Its Proliferation Output in Heart Development}

The Hippo pathway is a highly conserved mechanism for the regulation of heart size and maturation (Harvey et al., 2003; Pan, 2007; Hayashi et al., 2015). Its downstream transcriptional factors, YAP1/TAZ, are key regulators of embryonic and neonatal cardiac development, and this has been validated by multiple lines of evidence (Tables 2, 3). Mouse embryonic heart with SAV1 CKO $\left(N k \times 2.5^{\mathrm{cre}}: S A V^{\mathrm{f} / \mathrm{f}}\right)$ shows ventricular septal defect and abnormal heart growth, including thickening of ventricular walls, expansion of trabecular and ventricular myocardial layers, and enlargement of ventricular chambers (Heallen et al., 2011). Similarly, LATS2 and MST1/2 CKO hearts show the same abnormal heart phenotypes (Matsui et al., 2008; Heallen et al., 2011). The SAV CKO heart with reduced phosphorylation of YAP1 exhibit enhanced proliferation of CM and unchanged cell size (Heallen et al., 2011). A WWTR1 (or TAZ) deficiency in zebrafish heart results in arrested CM proliferation, reduced cardiac trabeculation, and immature trabecular bridges (Lai et al., 2018). YAP1 knockdown using Tnnt2-Cre in mouse fetal heart causes cardiac hypoplasia with reduced ventricular chamber size, ventricular septal defects, peripheral edema, and pericardial effusion; these embryos cannot survive beyond E16.5 (von Gise et al., 2012). YAP1 inactivation represses fetal CM proliferation; however, it does not affect the cell size. Consistent with this, YAP1 deletion in the postnatal heart using Tnnt2-Cre does not significantly affect physical cardiac hypertrophy (von Gise et al., 2012). Thus, the Hippo pathway regulates the normal cardiac structure and size by mediating the cell proliferation activities of YAP1/TAZ in the fetal and postnatal phase.

Yes-associated protein 1/TAZ, as vital effectors of the Hippo pathway, mainly affect target genes transcription and downstream signaling to exhibit their pro-proliferation activities in heart development (Table 3). Neuregulin 1 (Nrg1), secreted by endocardial cells, is a crucial factor in myocardial growth; it induces CM proliferation and re-entry of differentiated CMs 
TABLE 2 | Cardiac output of the Hippo-YAP1/TAZ pathway in cell and animal models.

\begin{tabular}{|c|c|c|c|c|}
\hline Gene & Model & & Promoter & Output \\
\hline \multirow[t]{10}{*}{ YAP1 } & Mouse & $\mathrm{CKO}$ & Tnnt2-Cre & $\begin{array}{l}\text { Cardiac hypoplasia (reduced ventricular chamber size, ventricular septal defects, peripheral } \\
\text { edema, and pericardial effusion) (von Gise et al., 2012) }\end{array}$ \\
\hline & Mouse & $\mathrm{CKO}$ & Nfatc 1 IRES-Cre/+ & $\begin{array}{l}\text { Less CM proliferation, impaired compact myocardium, and early postnatal lethality (Artap et al., } \\
\text { 2018) }\end{array}$ \\
\hline & Mouse & $\mathrm{CKO}$ & $\alpha-\mathrm{MHC}-\mathrm{Cre}$ & $\begin{array}{l}\text { Blunted cardiac hypertrophy and amplified CM apoptosis and fibrosis; cardiac dilatation and } \\
\text { dysfunction after TAC (Byun et al., 2019) }\end{array}$ \\
\hline & Mouse & $\mathrm{CKO}$ & Nkx2.5-cre & $\begin{array}{l}\text { Lack of healthy myocardial tissue in the left ventricle wall and an enhancing fibrotic infarct zone } \\
\text { following MI (Xin et al., 2013a) }\end{array}$ \\
\hline & Mouse & $\mathrm{CKO}$ & $\alpha-\mathrm{MHC}-\mathrm{Cre}$ & Thinned septal and posterior wall, and chamber dilation (Del Re et al., 2013). \\
\hline & Mouse & $\mathrm{CKO}$ & $\alpha-\mathrm{MHC}-\mathrm{Cre}$ & $\begin{array}{l}\text { Increasing CM apoptosis, fibrosis, enlarging infarct size, and impairing cardiac function (Del Re } \\
\text { et al., 2013) }\end{array}$ \\
\hline & Mouse & Overexpression & $\begin{array}{l}\text { Adeno- } \\
\text { associated } \\
\text { virus subtype 9: } \\
\text { human YAP1 }\end{array}$ & Alleviating MI injury and ameliorating cardiac function (Lin et al., 2014) \\
\hline & Mouse & $\begin{array}{l}\text { YAP5SA (active } \\
\text { YAP1) } \\
\text { overexpression }\end{array}$ & $\begin{array}{l}\text { aMyHC-Cre- } \\
\text { ERT2 }\end{array}$ & $\begin{array}{l}\text { Re-entering the cell cycle and reprogramming into more primitive and fetal cell states; thickened } \\
\text { ventricular walls and smaller chambers (Monroe et al., 2019) }\end{array}$ \\
\hline & Mouse & $\begin{array}{l}\text { YAPS112A } \\
\text { (active YAP1) } \\
\text { overexpression }\end{array}$ & $\alpha-\mathrm{MHC}$ & Increased myocardial tissue and reduced LV fibrosis in neonatal heart (Xin et al., 2013a) \\
\hline & $\begin{array}{l}\text { AC16 human } \\
\text { CMs }\end{array}$ & Overexpression & $\begin{array}{l}\text { Lentiviral } \\
\text { vectors }\end{array}$ & Reducing CM apoptosis, cell hypertrophy, and ROS generation after IR (Khan et al., 2019) \\
\hline WWTR1 (TAZ) & Zebrafish & $\mathrm{CKO}$ & CRISPR/CAS9 & $\begin{array}{l}\text { CM proliferation arrest, reduced cardiac trabeculation, and immature trabecular bridges (Lai } \\
\text { et al., 2018) }\end{array}$ \\
\hline WW45 & Mouse & $\mathrm{CKO}$ & Myh6-Cre & $\begin{array}{l}\text { Sustained YAP1 activation in CMs with cell-cycle re-entry, increased de-differentiation, and } \\
\text { decreased apoptosis; cardiac dysfunction, severe heart failure, and enhanced mortality in } \\
\text { response to TAC (Ikeda et al., 2019a,b) }\end{array}$ \\
\hline \multirow[t]{2}{*}{ SAV1 } & Mouse & $\mathrm{CKO}$ & Nkx2.5-cre & $\begin{array}{l}\text { Ventricular septal defect and abnormal heart growth (thickening of ventricular walls, expansion } \\
\text { of trabecular and ventricular myocardial layers, and enlargement of ventricular chambers) } \\
\text { (Heallen et al., 2011) }\end{array}$ \\
\hline & Mouse & $\mathrm{CKO}$ & $\begin{array}{l}\text { Myh6 } 6^{\text {CreERT2 }} \\
\alpha \mathrm{MHC}-\mathrm{mcm}\end{array}$ & $\begin{array}{l}\text { Presenting renewal capacity: increase in cell number and myocardial regeneration following } \\
\text { cardiac apex resection in postnatal hearts; increased LV CMs, less fibrosis, and improved } \\
\text { cardiac function after MI in adult heart (Heallen et al., 2013; Leach et al., 2017) }\end{array}$ \\
\hline \multirow[t]{2}{*}{ RASSF1A } & Mouse & $\begin{array}{l}\text { cardiomyocyte- } \\
\text { specific } \\
\mathrm{KO}\end{array}$ & $\alpha-\mathrm{MHC}-\mathrm{Cre}$ & $\begin{array}{l}\text { Basal nondistinctive cardiac phenotype or functional abnormality; reduced apoptosis, fibrosis, } \\
\text { and hypertrophy after TAC (Del Re et al., 2010) }\end{array}$ \\
\hline & Mouse & $\mathrm{KO}$ & NS & $\begin{array}{l}\text { Increased hypertrophic response, reduced cardiomyocytes apoptosis, and increased fibrosis } \\
\text { after TAC (Del Re et al., 2010) }\end{array}$ \\
\hline MST1/2 & Mouse & $\mathrm{CKO}$ & Nkx2.5-cre & Ventricular septal defect and abnormal heart growth (Heallen et al., 2011) \\
\hline \multirow[t]{2}{*}{ MST1 } & Mouse & $\begin{array}{l}\text { DN-MST1 } \\
\text { overexpression }\end{array}$ & CMV-Cre & $\begin{array}{l}\text { Reducing the size of Ml in the area at risk, and decreasing CM apoptosis (Nakamura et al., } \\
\text { 2016) }\end{array}$ \\
\hline & Mouse & $\begin{array}{l}\text { DN-MST1 } \\
\text { overexpression }\end{array}$ & $\alpha-\mathrm{MHC}$ & $\begin{array}{l}\text { Reduced left ventricular remodeling, improved left ventricular function, and enhanced survival } \\
\text { rate after Ml (Maejima et al., 2013) }\end{array}$ \\
\hline \multirow[t]{3}{*}{ LATS2 } & Mouse & $\begin{array}{l}\text { LATS2 } \\
\text { overexpression }\end{array}$ & $\alpha-\mathrm{MHC}$ & $\begin{array}{l}\text { Reduced left ventricular systolic and diastolic dysfunction, and smaller left/right ventricle (Matsui } \\
\text { et al., 2008) }\end{array}$ \\
\hline & Mouse & $\begin{array}{l}\text { DN-LATS2 } \\
\text { overexpression }\end{array}$ & $\alpha-\mathrm{MHC}$ & $\begin{array}{l}\text { Reduced CM apoptosis and enhanced biventricular hypertrophy following TAC (Matsui et al., } \\
\text { 2008) }\end{array}$ \\
\hline & Mouse & $\mathrm{CKO}$ & Nkx2.5-cre & Ventricular septal defect and abnormal heart growth (Heallen et al., 2011) \\
\hline \multirow[t]{2}{*}{ LATS1/2 } & Mouse & $\begin{array}{l}\text { LATS1/2 CKO; } \\
\text { YAP1/TAZ CKO }\end{array}$ & Wt ${ }^{\text {CreERT2 }}$ allele & Successful survival past E15.5 without defects in coronary vasculature (Xiao et al., 2018) \\
\hline & Mouse & $\mathrm{CKO}$ & Wt ${ }^{\text {CreERT2 }}$ allele & $\begin{array}{l}\text { Failing to survive past E15.5; smaller hearts with less compacted myocardium } \\
\text { (Xiao et al., 2018) }\end{array}$ \\
\hline
\end{tabular}


TABLE 2 | Continued

\begin{tabular}{|c|c|c|c|c|}
\hline Gene & Model & & Promoter & Output \\
\hline & Mouse & $\mathrm{CKO}$ & Myh6 $6^{\text {CreERT2 }}$ & $\begin{array}{l}\text { Presenting renewal capacity: increasing cell number and regenerating the myocardium following } \\
\text { cardiac apex resection in postnatal hearts; increased LV CMs, less fibrosis, and improved } \\
\text { cardiac function after MI in adult heart (Heallen et al., 2013) }\end{array}$ \\
\hline & Zebrafish & DKO & Myh6; Myl7 & Enhanced Hand2 expression and CM differentiation (Schindler et al., 2014; Fukui et al., 2018) \\
\hline
\end{tabular}

NS, not specified, where the specific promotors have not been validated; YAP1, Yes-associated protein 1; TAZ, PDZ-binding motif; CKO, cardiac knockout; KO, knockout; $D N$, dominant negative, MI, myocardial infarction; CM, cardiomyocytes; TAC, transverse aortic constriction; IR, ischemia-reperfusion; ROS, reactive oxygen species.

in the cell-cycle via Nrg1/ErbB2 signaling (Bersell et al., 2009; Gemberling et al., 2015). Targeting at Nrg1, YAP1/TAZ activates Nrg1/ErbB2 signaling in the endocardium; in addition, loss of YAP1 in the endocardium contributes to less CM proliferation, impaired development of compact myocardium, and early postnatal mortality (Artap et al., 2018). Moreover, Nrg1/ErbB4

TABLE 3 | Target genes and cardiac output of the Hippo-YAP1/TAZ pathway.

\begin{tabular}{|c|c|c|}
\hline Target genes & $\begin{array}{l}\text { Type of } \\
\text { Regulation }\end{array}$ & Outcome \\
\hline Bmp2b & Promotion & $\begin{array}{l}\text { Activating the BMP pathway to } \\
\text { enhance the number of CPCs in the } \\
\text { secondary heart field (Fukui et al., 2018) }\end{array}$ \\
\hline $\begin{array}{l}\text { Cyr61 and } \\
\text { CTGF }\end{array}$ & Promotion & $\begin{array}{l}\text { Promoting CM proliferation (Badouel } \\
\text { et al., 2015; Ragni et al., 2017; Zou } \\
\text { et al., 2018) }\end{array}$ \\
\hline $\begin{array}{l}\text { Dpp4 and } \\
\text { Dhrs3 }\end{array}$ & Promotion & $\begin{array}{l}\text { Inhibiting subepicardial cells from } \\
\text { differentiating into cardiac fibroblasts, } \\
\text { thus inducing coronary vessel defects } \\
\text { (Xiao et al., 2018) }\end{array}$ \\
\hline hand2 & Promotion & $\begin{array}{l}\text { Regulating differentiation of LPM cells } \\
\text { that develop into the atrium of the heart } \\
\text { (Fukui et al., 2018) }\end{array}$ \\
\hline miR-152 & Promotion & $\begin{array}{l}\text { Promoting CM proliferation (Wang } \\
\text { et al., 2018) }\end{array}$ \\
\hline miR-206 & Promotion & $\begin{array}{l}\text { Promoting cell hypertrophy and cell } \\
\text { survival by downregulating FoxP1 after } \\
\text { TAC (Yang et al., 2015) }\end{array}$ \\
\hline $\operatorname{Nrg} 1$ & Promotion & $\begin{array}{l}\text { Activating Nrg1/ErbB2 signaling and } \\
\text { reducing CM proliferation, to impair } \\
\text { compact myocardium development } \\
\text { (Artap et al., 2018). }\end{array}$ \\
\hline Oct-3/4 & Promotion & $\begin{array}{l}\text { Maintaining mouse and human } \\
\text { embryonic stem cell self-renewal } \\
\text { (Tamm et al., 2011) }\end{array}$ \\
\hline OSM & Promotion & $\begin{array}{l}\text { Regulating CM de-differentiation (Ikeda } \\
\text { et al., 2019a) }\end{array}$ \\
\hline Park2 & Promotion & $\begin{array}{l}\text { Promoting the clearance of impaired } \\
\text { mitochondria through autophagy, } \\
\text { subsequently enhancing CM resistance } \\
\text { to stress (Kubli et al., 2013; Leach } \\
\text { et al., 2017). }\end{array}$ \\
\hline Pik3cb & Promotion & $\begin{array}{l}\text { Activating PI3K-AKT signaling to trigger } \\
\text { CM proliferation and survival (Xin et al., } \\
\text { 2011; Lin et al., 2015) }\end{array}$ \\
\hline Sox17 & Inhibition & $\begin{array}{l}\text { Disrupting CM differentiation } \\
\text { (Hsu et al., 2018) }\end{array}$ \\
\hline
\end{tabular}

CM, cardiomyocytes; PI3K, phosphoinositol 3-kinase; TAC, transverse aortic constriction; LPM, lateral-plate mesoderm, CPC, cardiac precursor cell. signaling can reduce the phosphorylation of YAP1 Ser127, and promote YAP1-dependent CTGF transcription through the production of the ErbB4-YAP1-TEAD complex in breast cancer cells (Haskins et al., 2014). Thus, it is hypothesized that YAP1 proliferatively increases output not only by directly mediating CTGF transcription, but also indirectly by activating Nrg1/ErbB4 signaling. However, it is unclear whether Nrg1/ErbB4 signalingdependent YAP1 activation exists in the endocardium.

Through differential gene expression analysis, Pik3cb [which encodes a catalytic subunit of phosphoinositol-3-kinase (PI3K)] has been identified as the target gene of YAP1. YAP1 promotes $P i k 3 c b$ transcription to activate PI3K-AKT signaling and trigger CM proliferation and survival (Xin et al., 2011; Lin et al., 2015). In cases with Pik3cb deficiency, the mouse embryo cannot survive beyond E10.5 (Bi et al., 2002). Moreover, PI3K-AKT signaling inhibits glycogen synthase kinase $3 \beta$ (GSK3 $\beta$ )-dependent phosphorylation of $\beta$-catenin; the consequent activation of $\beta$-catenin promotes CM proliferation (Xin et al., 2011). The interaction between the Hippo pathway, Wnt/ $\beta$-catenin signaling, and PI3K-AKT signaling facilitates the proliferation of CMs. Besides the aforementioned signaling, YAP1/TAZ can also affect bone morphogenetic protein (BMP) pathway to regulate $\mathrm{CM}$ proliferation. By upregulating $B m p 2 b$ transcription, YAP1/TAZ activate the BMP pathway to enhance the number of cardiac precursor cells (CPCs) in the secondary cardiac field (Fukui et al., 2018). More importantly, BMP2 induces EMT in cardiac cushion, which facilitates the development of valves and septa (Gomez-Puerto et al., 2019). These mechanisms delineate the crosstalk between YAP1/TAZ and BMP pathway in cardiac development. Collectively, the above-mentioned findings suggest that the pro-proliferation activities of YAP1/TAZ participate in cardiac development from the fetal to postnatal periods, including the trabeculation and formation of the ventricles and endocardium. Thus, the functions of the Hippo pathway and YAP1/TAZ are shown to be highly conserved, as is the crosstalk between the Hippo pathway and other signaling pathways in cardiac development.

\section{Role of Hippo-YAP1/TAZ Signaling and Its Differentiation Output in Heart Development}

Besides cell proliferation, cardiogenesis involves cell differentiation; in addition, different cell types are involved in salient events during cardiac development. YAP1/TAZ are the key inhibitors of hESC differentiation into cardiac mesoderm. The CKO of LATS1/2 expression in the epicardium by using 
$\mathrm{Wt}^{\mathrm{CreERT} 2}$ prevents embryos from surviving past E15.5 (Xiao et al., 2018). Due to the deletion of LATS1/2, activated YAP1 inhibits the differentiation of subepicardial cells into cardiac fibroblasts which induces coronary vascular defects; however, the LATS1/2 CKO and YAP1/TAZ CKO embryos do not exhibit defects of the coronary vasculature. This indicates that LATS1/2 plays an important role in restricting YAP1/TAZ activities and that YAP1/TAZ are essential for normal heart growth (Xiao et al., 2018). In LATS1/2 CKO heart, downstream genes Dpp4 and Dhrs3 transcription is upregulated through the YAP1-TEAD complex, which regulates the differentiation events of subepicardial cells and the coronary vessel remodeling, respectively (Xiao et al., 2018). Dehydrogenase/reductase superfamily 3 (Dhrs3), a negative modulator of retinoic acid generation, inhibits retinoic acid signaling, which inhibits the differentiation of cardiac fibroblasts (Billings et al., 2013; Xiao et al., 2018). In response to LATS1/2 deficiency, Dipeptidyl peptidase-4 (Dpp4; a serine protease) alters the composition of the ECM and is involved in abnormal vessel development, such as increasing blood islands and defects of mean lacunarity (Ghersi et al., 2006; Xiao et al., 2018).

Furthermore, YAP1/TAZ regulate the transcription of other target genes to affect the progression of cell differentiation in cardiogenesis, such as Sox17 and hand2. Sox17, a member of SOX genes, plays a critical role in cardiac development. YAP1 selectively represses the expression of APS cell genes Sox17 to disrupt the differentiation of CMs (Hsu et al., 2018). Inhibition of YAP1 by dasatinib induces the differentiation of hESCs into APS-derived endoderm and cardiac mesoderm. In addition to cardiac mesoderm, Sox17 is also required for the development of endocardium. Sox17 deficiency leads to abnormal endocardium with impaired ventricular trabeculation and thickened myocardium (Lange et al., 2014). YAP1/TAZ, through facilitation of hand2 transcription, can regulate the differentiation of the lateral-plate mesoderm (LPM) cells, which finally develop into the atrium of the heart (Fukui et al., 2018). Hand2 can facilitate CM differentiation, and the expression of hand 2 can be potentiated by LATS1/2 double KO (DKO) (Schindler et al., 2014; Fukui et al., 2018). Therefore, YAP1/TAZ can facilitate de-differentiation and differentiation activity in different cell types during various phases of cardiac development.

\section{Regulation of YAP1/TAZ by Diverse Mechanisms in Heart Development}

During cardiac development, the content and activity of YAP1/TAZ are not always in a stable level. At approximately $\mathrm{P} 4$, myocardial cell numbers peak and this increase is reconciled with the percentage of nuclear YAP1 (Li et al., 1996). During the late maturation of trabeculae, CMs need to withdraw from the cell cycle and differentiate; if not, non-compaction cardiomyopathy occurs. YAP1 may modulate these events (Hertig et al., 2018). These studies indicate that during the early stage of development, there are high levels of YAP1/TAZ activity and abundance, contributing to cell proliferation and dedifferentiation; however, in the late stage, the proliferation effects of YAP1/TAZ are blunted for normal cardiac development, which underscores the importance of the spatiotemporal modulation of YAP1/TAZ. Thus, YAP1/TAZ activities need to be fine-tuned spatiotemporally depending upon the pace of cardiogenesis.

A diverse range of mechanisms are involved in the precise modulation of YAP1/TAZ activities in cardiac homeostasis and appropriate cardiac size. As noted previously, it is imperative to focus on the PTMs of YAP1/TAZ, as these affect the subcellular localization and activity of YAP1/TAZ. Previous studies have mainly shown that the phosphorylation of YAP1/TAZ is dependent on the canonical Hippo pathway. In the SAV1 CKO heart, YAP1 that is phosphorylated at Ser127 is reduced, thereby causing CM proliferation (Heallen et al., 2011). Moreover, Nrg1/ErbB4 signaling can inhibit LATS1-dependent phosphorylation at YAP1 Ser127 and promote YAP1-dependent CTGF transcription (Haskins et al., 2014). Cullin-RING ligases (CRLs), also known as the ubiquitin ligases, consist of Cullins, substrate-recognition, and RING proteins that mediate proteolysis of cellular proteins (Petroski and Deshaies, 2005). CRL-dependent ubiquitination appears to play an important role in temporal regulation of the Hippo-YAP1/TAZ signaling during cardiac development. In the developing heart, CRLs trigger the ubiquitination-induced degradation of MST1; this, in turn, induces dephosphorylation and nuclear translocation of YAP1, thereby ensuring CM proliferation (Zou et al., 2018). Nonetheless, the ubiquitination effect of CRLs depends on the neddylation of a ubiquitin-like protein, NEDD8. Consequently, cardiac-specific inhibition of NEDD8 attenuates the activity of CRLs and thereafter blunts the cell-proliferation activity of YAP1; this causes ventricular hypoplasia and non-compaction and, eventually, heart failure and neonatal death in mice (Zou et al., 2018).

In the developing heart, endogenous microRNAs (miRNAs) regulate the Hippo pathway and YAP1/TAZ to maintain heart homeostasis; miRNAs, small non-coding RNA molecules, silence mRNAs and suppress mRNA translation to control cardiac development (Ambros, 2004; Bartel, 2004). miR302-367 restrains MST1, LATS2, and MOB1b, which reduces the phosphorylation of YAP1 Ser127 and enhances the nuclear translocation of YAP1 (Tian et al., 2015). miR302-367, through activation of YAP1, promotes cell proliferation in embryonic and postnatal CMs (Tian et al., 2015). Using functional screen and computational approaches, some miRNAs were found to promote YAP1dependent $\mathrm{CM}$ proliferation by targeting and inhibiting the members of Hippo signaling (Tian et al., 2015; Diez-Cunado et al., 2018). For example, the predicted targets of miR-520d-3p are TAOK1/2 and LATS2; in addition, members of the miR-17 family repress TAOK1/2/3, MST2, SAV1, LATS2, MOBKL1A, and others (Diez-Cunado et al., 2018). Subsequently, miR520d-3p, miR-17 family and other detected miRNAs enhance the nuclear localization of YAP1 and induce pro-proliferation activity. However, inactivation of a single miRNA (such as miR520d-3p or miR-17 family) does not have any obvious effect on YAP1 activity; this suggests that none of these miRNAs can individually maintain YAP1 nuclear location and cell proliferation. This indicates that multiple endogenous miRNAs synergistically, but not individually, regulate Hippo signalinginduced cell proliferation during cardiac development. 
In the early postnatal period, cell-cell junctions in CMs progressively mature and modulate nuclear-cytoplasmic localization of YAP1/TAZ. The dystrophin-glycoprotein complex (DGC), a kind of transmembrane complex, serves as a link between the cellular cytoskeletal system and the ECM (Srivastava and Yu, 2006). DGC directly binds to YAP1 at the cell membrane, suppressing YAP1 activity. This interaction is synergistically augmented by the activation of the Hippo pathway; this suggests that Hippo signaling and DGC work in concert to inhibit the proliferation of murine CM (Morikawa et al., 2017). A cell junction protein Fat4 binds angiomotin-like 1 (AMOTL1, a isoform of angiomotin [AMOT] family) with YAP1 at the cell junction, leading to impaired cell proliferation in postnatal mouse heart; this is independent of the canonical Hippo pathway (Ragni et al., 2017). In the absence of Fat4, YAP1 translocates to the nucleus, promoting YAP1-dependent CTGF transcription; this leads to thicker ventricular myocardium and interventricular septum in the mouse heart with increased cell proliferation (Badouel et al., 2015; Ragni et al., 2017). Similar to this, AMOTL1 can bind to LATS2 at tight junction, and activate LATS2 to enhance YAP1 phosphorylation and repress YAP1 activity (Paramasivam et al., 2011). Therefore, cell junction related proteins may induce cytoplasmic sequestration of YAP1/TAZ; the consequent inhibition of YAP1 activity impairs heart growth after birth. Interestingly, AMOTL1 exhibits a dichotomous effect on the regulation of YAP1 activity. YAP1, forming a complex with AMOTL1, co-translocates to the nucleus and triggers proliferation activity as a result of Fat4 deficiency (Ragni et al., 2017); in addition, AMOTL1 inhibits YAP1 with the help of other junction proteins or the Hippo pathway (Paramasivam et al., 2011).

The above-mentioned studies reveal that the protein level and stability of YAP1/TAZ play a pivotal role in cardiac function and development. YAP1/YAZ imbalance leads to abnormal cardiac development, dysfunction, and even mortality. And YAP1/TAZ are mediated by several complex mechanisms such as PTMs, endogenous miRNAs, and cell-cell junctions, which makes it difficult to understand the complete molecular network and remains to be studied.

\section{ROLE OF HIPPO-YAP1/TAZ SIGNALING IN CARDIAC DISEASE}

The heart can be damaged by several stimuli such as inadequate nutrition, oxygen deficiency, and hemodynamic stress. Among these, ischemic heart disease plays a major role in cardiac disease. The regulation of the Hippo-YAP1/TAZ pathway appears to be cardiac protective, with relevant effects on cell proliferation, apoptosis, and cardiac remodeling (Tables 2, 3).

\section{Role of Hippo-YAP1/TAZ Signaling in Cardiac Ischemia-Reperfusion and Myocardial Infarction}

An increasing body of evidence has implicated YAP1/TAZ in the causation of cardiac ischemia-reperfusion (IR) damage.
IR induces activation of MST1 in mouse heart; however, after overexpressing dominant-negative MST1 (DN-MST1), the inactive MST1 induces reduction in myocardial infarction (MI) size in areas at risk of mouse heart (Nakamura et al., 2016). Endogenous MST1 potentiates IR-induced myocardial injury mainly by increasing apoptosis and partly by inhibiting autophagy, which is independent of the canonical Hippo pathway (Yamamoto et al., 2003; Nakamura et al., 2016). Mechanistically, the Ras-association domain family 1 isoform A (RASSF1A) enhances MST1 activity and translocation to the mitochondria; thereafter, activated MTS1 augments Baxor Bcl-xL-induced apoptosis and suppresses Beclin1-mediated autophagy (Yamamoto et al., 2003; Nakamura et al., 2016). In this setting, CM autophagy, as a protective mechanism, degrades and recycles harmful cytoplasmic components, which contributes to repair of IR damage and promotes cell survival; in contrast, apoptosis was shown to perform an anti-survival role in IR damage (Nishida et al., 2008). Similar results have been observed in models of MI. After MI, endogenous MST1 potentiates left ventricular (LV) enlargement, impairs LV ejection fraction, and reduces the survival rate by suppressing autophagy (Odashima et al., 2007; Maejima et al., 2013). MImediated MST1 activation stabilizes the Beclin1 homodimer, and suppresses the downstream output of Beclin1 to inhibit autophagy; this impairs the recycling of harmful components and protein quality control (Maejima et al., 2013). Besides the MST1Bax-Bcl-xL axis, MST1 mediates the canonical Hippo pathway to exert pro-apoptotic activity. In cultured CMs, LATS2 was shown to increase cell apoptosis in a dose-dependent manner; in contrast, dominant-negative LATS2 was found to suppress MST1-induced apoptosis (Matsui et al., 2008). IR induces activation of NF2 and potentiation of MST1 activity, which increases CM apoptosis; hearts lacking NF2 by Cre ${ }^{\alpha M H C}$ exhibit diminished phosphorylation of MST1 and activation of YAP1 (Matsuda et al., 2016). In the NF2 CKO heart, enhanced YAP1 activity protects against IR damage. Collectively, these findings suggest that MST1 participates in IR and MI injury by enhancing apoptosis and reducing autophagy; moreover, MST1 works as a negative regulator in cardiac protection through Hippodependent or -independent mechanisms. Based on the available evidence, it can be indirectly inferred that the Hippo pathway may be an anti-survival mediator in cardiac IR and MI damage.

As mentioned previously, IR and MI stress induce activation of the Hippo pathway and inactivation of YAP1/TAZ. Activated YAP1 has a protective effect on IR-damaged heart. Lentivirusgenerated YAP1 overexpression in AC16 human CMs decreases $\mathrm{CM}$ apoptosis, cell hypertrophy, and generation of reactive oxygen species (ROS), which protects CMs from IR injury (Khan et al., 2019). Interestingly, downregulation of the hypertrophy effect of YAP1 is not consistent with the results of previous studies that showed that YAP1 promotes CM hypertrophy after IR stress (Yang et al., 2015; Zhou et al., 2015). This downregulation of the hypertrophy effect may be secondary to decreased apoptosis and ROS generation instead of direct regulation by YAP1; therefore, there exists a difference. Several mechanisms explain the cardioprotective function of YAP1. YAP1 inhibits the ataxia-telangiectasia 
mutated (ATM)/ATM-and Rad3-related (ATR) DNA-damage cascade to decrease apoptosis (Khan et al., 2019). ATM and ATR are sensors of DNA lesions that recognize failed DNA repairs and excessive DNA lesions and consequently trigger apoptosis (Roos and Kaina, 2013). In addition, YAP1 mediates mitochondrial homeostasis to resist ischemic stress. YAP1 can activate optic atrophy 1 (OPA-1)-mediated mitochondrial fusion, which ensures mitochondrial homeostasis (Ma and Dong, 2019). Mitochondrial fusion can reduce IR-induced mitochondrial fragmentation and mitochondrial oxidative stress, and suppress mitochondrial apoptosis; this promotes cell survival and alleviates cardiac IR injury (Anzell et al., 2018; Ma and Dong, 2019). Park2 (a YAP1 target gene) encodes Parkin, which is an important E3 ubiquitin ligase in the outer mitochondrial membrane. Parkin promotes the clearance of impaired mitochondria through mitochondrial autophagy, which enhances stress resistance of CMs (Kubli et al., 2013; Leach et al., 2017). Moreover, YAP1 binding to Forkhead box protein O1 (FoxO1) was shown to facilitate antioxidant catalase and manganese superoxide dismutase transcription; the consequent decrease in ROS generation was shown to protect CMs against IR-induced oxidative stress (Shao et al., 2014). In the setting of MI, heterozygous inactivation of YAP1 in heart enhances CM apoptosis, fibrosis, enlarges infarct size, and impairs cardiac function; YAP1 activation using adeno-associated virus subtype 9: human YAP1 (AAV9: hYAP1) can alleviate MI injury and ameliorate cardiac function (Lin et al., 2014). In contrast to IR, YAP1 exerts anti-apoptosis activity via activation of PI3K-AKT signaling and increases $\mathrm{CM}$ proliferation against MI damage. Therefore, YAP1 mainly suppresses cell apoptosis through several downstream pathways and outputs to protect CMs and the heart from IR and MI damage.

\section{Role of Hippo-YAP1/TAZ Signaling in Cardiac Pressure Overload}

Similar to IR damage, endogenous RASSF1A phosphorylates and activates MST1 to promote CM apoptosis and impair cardiac function following transverse aortic constriction (TAC) - a form of PO (Del Re et al., 2010). This RASSF1A-induced apoptosis can be inhibited by MST1 deficiency. In the heart with CM-specific KO of RASSF1A, endogenous MST1 activation is abolished, and there is reduced apoptosis, fibrosis, and hypertrophy after TAC (Del Re et al., 2010). Thus, the RASSF1A-MST1 pathway is implicated in causing heart damage in response to $\mathrm{PO}$ stress. Another component of the Hippo pathway, LATS2, is elevated and activated after TAC. Cardiac overexpression of LATS2 was shown to be associated with diminished left ventricular systolic and diastolic dysfunction and smaller left and right ventricles (Matsui et al., 2008). Overexpression of dominant negative LATS2 (DN-LATS2) in the murine heart was found to repress CM apoptosis and promote hypertrophy following TAC (Matsui et al., 2008). In addition, a recent study showed activation of endogenous YAP1 (the terminal effector of the Hippo pathway) in the compensated phase in response to TAC (Byun et al., 2019). Heterozygous cardiacspecific YAP1 KO mice, when subjected to TAC, present blunted cardiac hypertrophy and amplified CM apoptosis and fibrosis, with resultant cardiac dilatation and dysfunction (Byun et al., 2019). The Hippo pathway, as a deleterious regulator, has been shown to exacerbate PO-induced cardiac damage; therefore, inhibition of the Hippo pathway may have a cardioprotective effect against PO injury. Mechanistically, YAP1 directly triggers the transcription of target genes to mediate downstream output and to protect the heart against PO damage. miR-206 is identified as the novel target gene of YAP1; thus, YAP1induced miR-206 expression promotes cell hypertrophy and cell survival by downregulating the expression of the Forkhead box protein P1 (FoxP1) (Yang et al., 2015). YAP1, through its regulation of miR-206, promotes cell hypertrophy and exerts a cardioprotective effect in response to TAC. Moreover, YAP1 promotes $P i k 3 c b$ transcription to activate PI3K-AKT signaling and trigger CM proliferation and survival (Xin et al., 2011; Lin et al., 2015). Collectively, these studies demonstrate the cardioprotective role of YAP1/TAZ against several stressors. Thus, YAP1/TAZ may serve as a therapeutic target in many cardiac diseases.

\section{Pernicious Role of YAP1/TAZ in Cardiac Disease}

Although YAP/TAZ undertake the cardioprotective role against cardiac damage, high levels of YAP1/TAZ activity are not always beneficial. The effects of long-term suppression of Hippo signaling or activation of YAP1/TAZ in cardiac disease are opposite to those of short-term suppression. Clinical ischemic heart disease and idiopathic dilated cardiomyopathy (DCM) is characterized by enhanced YAP1/TAZ protein levels and increased transcriptional activity with the resultant upregulation of target genes, such as ankyrin repeat domain 1, CTGF, and CYR61; moreover, this phenomenon was observed in the murine heart with desmin-related cardiomyopathy (Hou et al., 2017). The WW45 CKO by Myh6-Cre mice show sustained YAP1 activation in CMs, which induces cardiac dysfunction, severe heart failure, and enhanced mortality in response to TAC (Ikeda et al., 2019a,b). CMs are subject to cell-cycle reentry, increased de-differentiation, and decreased apoptosis; however, heart with active YAP1 cannot be rescued from PO damage (Ikeda et al., 2019a,b). This may be explained by the dual role of oncostatin M (OSM), which is identified as a novel target of the YAP1-TEAD1 complex. OSM promotes $\mathrm{CM}$ de-differentiation and induces the expression of stem cell markers to improve CM resistance to damage; however, excessive OSM stimulation inhibits CM contractility and induces cardiac remodeling (Kubin et al., 2011). In addition, YAP1-dependent OSM expression further activates YAP1 by inhibiting LATS2induced phosphorylation, thereby forming a positive feedback loop (Ikeda et al., 2019a). This positive feedback mechanism results in sustained YAP1 activation and redundant OSM, which exacerbates cardiac injury and heart failure upon PO. Furthermore, the YAP1/TEAD1-OSM feedback cycle develops in the heart of high-fat-diet (HFD)-fed mice following PO, and contributes to the progression of diabetic cardiomyopathy (Ikeda et al., 2019b). 
These observations suggest a pleiotropic role of YAP1 (Ikeda et al., 2019b). YAP1 activation during the acute phase of $\mathrm{CM}$ injury can induce compensatory cell proliferation and dedifferentiation, which temporarily improves the tolerance of $\mathrm{CM}$ to damage. In later periods, persistent activation of YAP1 produces cardiac dysfunction and heart failure, which may be partly caused by the YAP1/TEAD1-OSM positive feedback loop and cardiac remodeling. However, these events do not completely explain the cardiac pathology, and there is a need to further explore the underlying mechanisms. The available evidence suggests the significance of sustaining the appropriate YAP1/TAZ protein level in therapy of cardiac disease.

The involvement of the Hippo pathway in cardiac fibrosis and remodeling explains the pernicious role of YAP1/TAZ in cardiac disease. In a murine DCM model, endogenous Angiotensin II (Ang II) was shown to activate YAP1 and promote the proliferation of cardiac fibroblasts and their transdifferentiation to myofibroblasts; subsequently, these changes were found to induce cardiac remodeling and impaired cardiac contractility (Jin et al., 2019). The Hippo-YAP1/TAZ pathway, as a mechanosensor, is affected by mechanical signaling through cell geometry and alterations in cytoskeletal tension; this may help elucidate the pathological mechanism of chronic heart failure (Dupont et al., 2011). The subcellular localization and activity of YAP1/TAZ varies in cells with different matrix stiffness. In a stiff matrix, there is nuclear translocation and higher transcriptional activity of YAP1/TAZ; in contrast, a soft matrix is characterized by cytoplasmic sequestration and reduced activity of YAP1/TAZ (Nasrollahi and Pathak, 2017). The above studies potentially support the hypothesis that, in the context of cardiac ECM remodeling and/or fibrosis, the ECM stiffness and/or cytoskeletal tension may change and affect YAP1 activity; furthermore, activated YAP1 modulates the progression of ECM remodeling and fibrosis, which further exacerbates cardiac injury and even contributes to the development of chronic heart failure. However, the relationship between mechanical stimuli, remodeling/fibrosis, and YAP1/TAZ is not well characterized; therefore, studies to identify YAP1/TAZ activity and effects in cardiac disease are urgently needed.

\section{ROLE OF HIPPO-YAP1/TAZ SIGNALING IN CARDIAC REGENERATION}

Several endogenous and exogenous factors may cause cardiac damage and induce cardiac dysfunction. The heart is a highly differentiated organ with limited regenerative activity; this makes it difficult to restore normal function after any injury (Moya and Halder, 2018). Moreover, the regenerative capability of the adult heart is less than that of the neonatal heart. Consequently, cardiac regeneration can be activated through re-entry of terminally differentiated cells into cell cycle, reactivation of cell proliferation, and/or differentiation of stem cells into CMs, which contributes to post-damage recovery (Tables 2, 3; Ponnusamy et al., 2017; Moya and Halder, 2018).

As a pathway that controls organ size, Hippo-YAP1/TAZ signaling is essential for cardiac regeneration and could be applied for cardiac regeneration and targeted therapeutics in cardiac disease. The downstream effectors of YAP1/TAZ have clear biological effects in cardiac regeneration and CM protection. Studies have shown that adult and postnatal hearts with the deletion of SAV or LATS1/2 exhibit renewal capacity, which contributes to recovery after MI and cardiac apex resection (Heallen et al., 2013; Leach et al., 2017). Postnatal hearts lacking Hippo exhibit increase in cell number and myocardial regeneration following cardiac apex resection; postMI, SAV CKO hearts show increased LV CMs, less fibrosis, and improved cardiac function (Heallen et al., 2013; Leach et al., 2017). Mechanically, SAV deletion was found to upregulate cell cycle genes and heart growth-related genes, which increases DNA synthesis and induces cell-cycle re-entry (Leach et al., 2017). Among these upregulated genes, Park2 (encoding Parkin) was found to promote cell regeneration following stress. Parkin participates in the clearance of damaged mitochondria through mitochondria autophagy and apoptosis, and decreases the CM sensitivity to ischemic stress (Kubli et al., 2013; Leach et al., 2017). Additionally, studies have demonstrated enhanced expression of Paired-like homeodomain transcription factor 2 (Pitx2) in the Hippo-deficient heart (Tao et al., 2016). Pitx2, as a transcriptional factor, has the capacity to regulate several target genes encoding mitochondrial, oxidation-reduction, and respiratory chain proteins via binding to YAP1 or other undefined co-factors. Therefore, Pitx2 and YAP1 may synergistically trigger anti-oxidative effect and proliferation activity to improve the stress resistance and regeneration of adult and neonatal heart (Shao et al., 2014; Tao et al., 2016). These findings suggest that the Hippo pathway plays the role of a pernicious mediator in the context of cardiac regeneration.

The YAP1 CKO neonatal heart lacks healthy myocardial tissue in the LV wall and shows enhanced fibrotic infarct zone following an $\mathrm{MI}$; neonatal heart expressing active form of YAP1 exhibits increased myocardial tissue and reduced LV fibrosis (Xin et al., 2013a). Thus, the proliferative activity of YAP1 is essential for cardiac regeneration, and its regenerative activity is involved in proliferative gene programs. Mechanically, glycolytic YAP1 activation upregulates miR-152 expression to increase CM proliferation in neonatal mice (Wang et al., 2018). Furthermore, miR-152 suppresses the expression of cell-cycle inhibitory proteins, such as p27kip and DNA methyltransferase1 (DNMT1), leading to re-entry of neonatal CMs in the cell cycle. YAP1 activation promotes cell proliferation not only directly through targeting of the CTGF but also indirectly through inhibition of cell-cycle-inhibitory proteins; this contributes to neonatal cardiac regeneration and repair after MI injury (Haskins et al., 2014; Wang et al., 2018). As mentioned in Section "Role of Hippo-YAP1/TAZ Signaling and Its Differentiation Output in Heart Development," YAP1/TAZ have the capacity to induce proliferation and de-differentiation through targeting of the related genes (Pik3cb, Dpp4,Dhrs3, SOX17, Bmp2b, and others), which may induce CMs to re-enter the cell cycle and regeneration (Billings et al., 2013; Khurana et al., 2013; Lin et al., 2015; Estaras et al., 2017; Fukui et al., 2018; Hsu et al., 2018; Xiao et al., 2018). 
In addition to their action on highly differentiated CMs, YAP1/TAZ regulate stem cells to undergo differentiation and proliferation to achieve cardiac regeneration. $\mathrm{P}_{2} \mathrm{Y}_{2}$ receptor $\left(\mathrm{P} 2 \mathrm{Y}_{2} \mathrm{R}\right)$ is a pro-regenerative GPCR that regulates cell regeneration after damage (Vassort, 2001). Activated $\mathrm{P} 2 \mathrm{Y}_{2} \mathrm{R}$ promotes the proliferation and migration of human $\mathrm{c}-\mathrm{Kit}^{+}$ cardiac progenitor cells (hCPCs) via activation of YAP1 (Khalafalla et al., 2017). Moreover, hCPCs improves cardiac function in MI animal models, which supports the use of hCPCs for stem cell therapy in cardiac failure (Ellison et al., 2013). YAP5SA, an active version of YAP1, is overexpressed in the mouse heart. YAP5SA overexpression causes CMs to partially re-enter the cell cycle; in addition, CMs can be reprogrammed into a more primitive and fetal cell state, whereby the heart exhibits thickened ventricular walls and smaller chambers (Monroe et al., 2019). Active Yes phosphorylates YAP1 on one or more tyrosine residues; subsequently, phosphorylated YAP1 translocates into the nucleus and induces the transcription of the key pluripotency factor Oct-3/4 (Tamm et al., 2011). Oct$3 / 4$ is critical for maintaining the self-renewal capacity of mouse and human ESCs. It promotes the cell cycle re-entry of ESCs via suppressing the transcription of cyclin-dependent kinase inhibitors (Lee et al., 2010). These findings indicate that YAP1 plays a critical role in maintaining the proliferative and dedifferentiation capacity of stem cells and promoting adult CM renewal following injury.

Yes-associated protein 1/TAZ have the capacity to reactivate $\mathrm{CM}$ proliferation and may play a role in cardiac regeneration and therapy for cardiac disease. Nonetheless, the complete mechanism of action and the effects of YAP1/TAZ in cardiac development and disease remain an enormous puzzle. Therefore, further research is required to unravel the contributory mechanisms in order to clearly understand the therapeutic potential of targeted activation of the YAP1/TAZ signaling pathway in myocardial disease and cardiac regeneration.

\section{COMPOUNDS THAT REGULATE HIPPO-YAP1/TAZ SIGNALING}

Several reports have focused on the role of Hippo-YAP1/TAZ signaling in cardiac disease to explore the efficacy of leading therapeutic compounds and agents. The YAP1/TAZ-TEAD complex is considered a novel drug target to affect its biological output. Verteporfin, an inhibitor of YAP1, has been widely used to study the Hippo-YAP1/TAZ pathway (Khalafalla et al., 2017; Ikeda et al., 2019b). Verteporfin combined with YAP1 prevents the interaction of the YAP1-TEADs complex; verteporfin alters the conformation of YAP1 to enhance its binding with trypsin as well as tryptic cleavage (Liu-Chittenden et al., 2012; Tang et al., 2019). As mentioned in Section "Role of HippoYAP1/TAZ Signaling and Its Differentiation Output in Heart Development," YAP1/TEAD1-OSM feedback cycle exacerbates heart failure and the progression of diabetic cardiomyopathy in HFD-fed mice following TAC (Ikeda et al., 2019b). Verteporfin treatment via suppressing YAP1/TEAD1 complex alleviates cardiac dysfunction and improves the survival rate of HFD-fed mice by increasing the expression of CM dedifferentiation protein and by attenuating myocardial infiltration. $\mathrm{P} 2 \mathrm{Y}_{2} \mathrm{R}$ induced YAP1 activation promotes the proliferation and migration of hCPCs, which can be repressed by verteporfin (Khalafalla et al., 2017). Therefore, verteporfin may abolish the capacity of hCPCs in repairing MI injury (Ellison et al., 2013). YAP1/TAZ luciferase reporter assays have identified apigenin as a potential YAP1 inhibitor; it was found to disrupt the interaction between YAP1 and TEAD (Li et al., 2018b). Apigenin, a kind of flavonoid, was found to exhibit a protective effect against cardiovascular diseases. In vivo and vitro, apigenin suppresses ROS production, loss of mitochondrial membrane potential (MMP), and apoptosis via PI3K/AKT signaling as well as mitochondrial Notch1/Hes1 signaling; this was found to protect H9C2 cells and rat hearts against IR injury (Hu et al., 2015; Zhou et al., 2018). However, there is no evidence to suggest that apigenin can mediate the Hippo-YAP1/TAZ pathway to defend heart against stress.

Yes-associated protein 1/TAZ are affected by other pathways; in addition, certain drugs have been shown to target the upstream regulation of YAP1. Dasatinib, which targets the mitogen-activated protein kinase (MAPK) pathway (a proproliferative pathway, in contrast to Hippo signaling), may affect YAP1 phosphorylation and inhibit its activity (Rosenbluh et al., 2012; He et al., 2016). Inhibition of YAP1 by dasatinib was found to induce differentiation of hESCs into APS-derived endoderm and cardiac mesoderm, consequently affecting the progression of cell differentiation in cardiogenesis (Hsu et al., 2018). GPCRs are targeted by extracellular ligands and regulate YAP1 activity. As mentioned in Section "Role of Hippo-YAP1/TAZ Signaling and Its Differentiation Output in Heart Development," Ang II and melatonin activate YAP1 through GPCRs to induce corresponding changes in cardiac function (Jin et al., 2019; Ma and Dong, 2019). YAP1 is activated by endogenous Ang II to promote the proliferation of cardiac fibroblasts and their transdifferentiation to myofibroblasts, inducing cardiac remodeling and DCM (Jin et al., 2019). In addition, melatonin facilitates OPA-1mediated mitochondrial fusion via activating YAP1, which attenuates IR-induced mitochondrial apoptosis and alleviates cardiac IR damage (Ma and Dong, 2019). Dobutamine enhances YAP1 Ser127 phosphorylation and cytoplasmic sequestration through $\beta$-adrenergic receptor (a class of GPCRs) rather than the Hippo pathway (Bao et al., 2011). A35 (an antitumor compound) induces mutation of YAP1 Ser127, which induces the recovery of proliferative inhibition and apoptosis compared to wild-type YAP1 (Zhao et al., 2018). Although Dobutamine and A35 can mediate YAP activity in cancer cells, their effects on YAP1 in CMs and heart tissue are yet to be elucidated.

In conclusion, drugs and compounds that control kinases upstream and/or downstream of YAP1/TAZ can regulate YAP1/TAZ activity and subsequently influence cardiac injury. Undoubtedly, YAP1/TAZ is a potential therapeutic target for cancer and cardiac disease. However, the latent regulatory network of YAP1/TAZ has not been completely elucidated in previous research, especially in the context of cardiac disease. 


\section{AUTHOR CONTRIBUTIONS}

$\mathrm{NH}, \mathrm{XC}$, and YL participated in research design. $\mathrm{NH}$ and JL supervised in research design. XC and YL performed the data analysis. $\mathrm{NH}, \mathrm{JL}, \mathrm{XC}$, and YL wrote or contributed to the writing of the manuscript.

\section{REFERENCES}

Ambros, V. (2004). The functions of animal microRNAs. Nature 431, 350-355. doi: $10.1038 /$ nature02871

Anorga, S., Overstreet, J. M., Falke, L. L., Tang, J., Goldschmeding, R. G., Higgins, P. J., et al. (2018). Deregulation of Hippo-TAZ pathway during renal injury confers a fibrotic maladaptive phenotype. FASEB J. 32, 2644-2657. doi: 10.1096/ fj.201700722R

Anzell, A. R., Maizy, R., Przyklenk, K., and Sanderson, T. H. (2018). Mitochondrial quality control and disease: insights into ischemia-reperfusion injury. Mol. Neurobiol. 55, 2547-2564. doi: 10.1007/s12035-017-0503-9

Artap, S., Manderfield, L. J., Smith, C. L., Poleshko, A., Aghajanian, H., See, K., et al. (2018). Endocardial Hippo signaling regulates myocardial growth and cardiogenesis. Dev. Biol. 440, 22-30. doi: 10.1016/j.ydbio.2018.04.026

Badouel, C., Zander, M. A., Liscio, N., Bagherie-Lachidan, M., Sopko, R., Coyaud, E., et al. (2015). Fat1 interacts with Fat4 to regulate neural tube closure, neural progenitor proliferation and apical constriction during mouse brain development. Development 142, 2781-2791. doi: 10.1242/dev.123539

Bao, Y., Nakagawa, K., Yang, Z., Ikeda, M., Withanage, K., Ishigami-Yuasa, M., et al. (2011). A cell-based assay to screen stimulators of the Hippo pathway reveals the inhibitory effect of dobutamine on the YAP-dependent gene transcription. J. Biochem. 150, 199-208. doi: 10.1093/jb/mvr063

Bartel, D. P. (2004). MicroRNAs: genomics, biogenesis, mechanism, and function. Cell 116, 281-297.

Ben Mimoun, S., and Mauviel, A. (2018). Molecular mechanisms underlying TGFbeta/Hippo signaling crosstalks - role of baso-apical epithelial cell polarity. Int. J. Biochem. Cell Biol. 98, 75-81. doi: 10.1016/j.biocel.2018.03.006

Bersell, K., Arab, S., Haring, B., and Kuhn, B. (2009). Neuregulin1/ErbB4 signaling induces cardiomyocyte proliferation and repair of heart injury. Cell 138, $257-$ 270. doi: 10.1016/j.cell.2009.04.060

Bi, L., Okabe, I., Bernard, D. J., and Nussbaum, R. L. (2002). Early embryonic lethality in mice deficient in the p110beta catalytic subunit of PI 3-kinase. Mamm. Genome 13, 169-172. doi: 10.1007/bf02684023

Billings, S. E., Pierzchalski, K., Tjaden, N. E. B., Pang, X. Y., Trainor, P. A., Kane, M. A., et al. (2013). The retinaldehyde reductase DHRS3 is essential for preventing the formation of excess retinoic acid during embryonic development. Faseb. J. 27, 4877-4889. doi: 10.1096/fj.13-227967

Byun, J., Del Re, D. P., Zhai, P., Ikeda, S., Shirakabe, A., Mizushima, W., et al. (2019). Yes-associated protein (YAP) mediates adaptive cardiac hypertrophy in response to pressure overload. J. Biol. Chem. 294, 3603-3617. doi: 10.1074/jbc. RA118.006123

Chang, L., Azzolin, L., Di Biagio, D., Zanconato, F., Battilana, G., Xiccato, R. L., et al. (2018). The SWI/SNF complex is a mechanoregulated inhibitor of YAP and TAZ. Nature 563, 265-269. doi: 10.1038/s41586-018-0658-1

Cho, H., Kim, J., Ahn, J. H., Hong, Y. K., Makinen, T., Lim, D. S., et al. (2019). YAP and TAZ negatively regulate Prox1 during developmental and pathologic lymphangiogenesis. Circ. Res. 124, 225-242. doi: 10.1161/CIRCRESAHA.118. 313707

Cho, Y. S., Zhu, J., Li, S., Wang, B., Han, Y., and Jiang, J. (2018). Regulation of Yki/Yap subcellular localization and Hpo signaling by a nuclear kinase PRP4K. Nat. Commun. 9:1657. doi: 10.1038/s41467-018-04090-2

Dai, X., Liu, H., Shen, S., Guo, X., Yan, H., Ji, X., et al. (2017). YAP activates the Hippo pathway in a negative feedback loop. Cell Res. 27:1073. doi: 10.1038/cr. 2017.96

Del Re, D. P., Matsuda, T., Zhai, P., Gao, S., Clark, G. J., Van Der Weyden, L., et al. (2010). Proapoptotic Rassf1A/Mst1 signaling in cardiac fibroblasts is protective against pressure overload in mice. J. Clin. Invest. 120, 3555-3567. doi: $10.1172 /$ JCI43569

\section{FUNDING}

This work was supported by the National Natural Science Foundation of China (grant Nos. 81773720, 81402928, and 81573433) and Guangzhou Municipal Science and Technology Project (grant No. 201804010490).

Del Re, D. P., Yang, Y., Nakano, N., Cho, J., Zhai, P., Yamamoto, T., et al. (2013). Yes-associated protein isoform 1 (Yap1) promotes cardiomyocyte survival and growth to protect against myocardial ischemic injury. J. Biol. Chem. 288, 3977-3988. doi: 10.1074/jbc.M112.436311

Diez-Cunado, M., Wei, K., Bushway, P. J., Maurya, M. R., Perera, R., Subramaniam, S., et al. (2018). miRNAs that induce human cardiomyocyte proliferation converge on the hippo pathway. Cell Rep. 23, 2168-2174. doi: 10.1016/j.celrep. 2018.04.049

Dong, J., Feldmann, G., Huang, J., Wu, S., Zhang, N., Comerford, S. A., et al. (2007). Elucidation of a universal size-control mechanism in Drosophila and mammals. Cell 130, 1120-1133. doi: 10.1016/j.cell.2007.07.019

Dupont, S., Morsut, L., Aragona, M., Enzo, E., Giulitti, S., Cordenonsi, M., et al. (2011). Role of YAP/TAZ in mechanotransduction. Nature 474, 179-183. doi: 10.1038 /nature10137

Ellison, G. M., Vicinanza, C., Smith, A. J., Aquila, I., Leone, A., Waring, C. D., et al. (2013). Adult c-kit(pos) cardiac stem cells are necessary and sufficient for functional cardiac regeneration and repair. Cell 154, 827-842. doi: 10.1016/j. cell.2013.07.039

Elosegui-Artola, A., Andreu, I., Beedle, A. E. M., Lezamiz, A., Uroz, M., Kosmalska, A. J., et al. (2017). Force triggers YAP nuclear entry by regulating transport across nuclear pores. Cell 171, 1397-1410.e14. doi: 10.1016/j.cell.2017.10.008

Estaras, C., Hsu, H.-T., Huang, L., and Jones, K. A. (2017). YAP repression of the WNT3 gene controls hESC differentiation along the cardiac mesoderm lineage. Genes Dev. 31, 2250-2263. doi: 10.1101/gad.307512.117

Fang, L., Teng, H., Wang, Y., Liao, G., Weng, L., Li, Y., et al. (2018). SET1Amediated mono-methylation at K342 regulates YAP activation by blocking its nuclear export and promotes tumorigenesis. Cancer Cell 34, 103-118.e9. doi: 10.1016/j.ccell.2018.06.002

Fukui, H., Miyazaki, T., Chow, R. W.-Y., Ishikawa, H., Nakajima, H., Vermot, J., et al. (2018). Hippo signaling determines the number of venous pole cells that originate from the anterior lateral plate mesoderm in zebrafish. eLife 7:e29106. doi: 10.7554/eLife.29106

Garbino, A., Van Oort, R. J., Dixit, S. S., Landstrom, A. P., Ackerman, M. J., and Wehrens, X. H. (2009). Molecular evolution of the junctophilin gene family. Physiol. Genomics 37, 175-186. doi: 10.1152/physiolgenomics.00017.2009

Gemberling, M., Karra, R., Dickson, A. L., and Poss, K. D. (2015). Nrg1 is an injury-induced cardiomyocyte mitogen for the endogenous heart regeneration program in zebrafish. eLife 4:e05871. doi: 10.7554/eLife.05871

Ghersi, G., Zhao, Q., Salamone, M., Yeh, Y., Zucker, S., and Chen, W. T. (2006). The protease complex consisting of dipeptidyl peptidase IV and seprase plays a role in the migration and invasion of human endothelial cells in collagenous matrices. Cancer Res. 66, 4652-4661. doi: 10.1158/0008-5472.can-05-1245

Gomez-Puerto, M. C., Iyengar, P. V., Garcia De Vinuesa, A., Ten Dijke, P., and Sanchez-Duffhues, G. (2019). Bone morphogenetic protein receptor signal transduction in human disease. J. Pathol. 247, 9-20. doi: 10.1002/path.5170

Harvey, K. F., Pfleger, C. M., and Hariharan, I. K. (2003). The Drosophila Mst ortholog, hippo, restricts growth and cell proliferation and promotes apoptosis. Cell 114, 457-467. doi: 10.1016/s0092-8674(03)00557-9

Harvey, N. L., Srinivasan, R. S., Dillard, M. E., Johnson, N. C., Witte, M. H., Boyd, K., et al. (2005). Lymphatic vascular defects promoted by Prox1 haploinsufficiency cause adult-onset obesity. Nat. Genet. 37, 1072-1081. doi: $10.1038 /$ ng 1642

Haskins, J. W., Nguyen, D. X., and Stern, D. F. (2014). Neuregulin 1-activated ERBB4 interacts with YAP to induce Hippo pathway target genes and promote cell migration. Sci. Signal. 7:ra116. doi: 10.1126/scisignal.2005770

Hayashi, S., Yokoyama, H., and Tamura, K. (2015). Roles of Hippo signaling pathway in size control of organ regeneration. Dev. Growth Differ. 57, 341-351. doi: $10.1111 /$ dgd.12212 
He, M., Zhou, Z., Shah, A. A., Hong, Y., Chen, Q., and Wan, Y. (2016). New insights into posttranslational modifications of Hippo pathway in carcinogenesis and therapeutics. Cell Div. 11:4. doi: 10.1186/s13008-016-0013-6

Heallen, T., Morikawa, Y., Leach, J., Tao, G., Willerson, J. T., Johnson, R. L., et al. (2013). Hippo signaling impedes adult heart regeneration. Development 140 , 4683-4690. doi: 10.1242/dev.102798

Heallen, T., Zhang, M., Wang, J., Bonilla-Claudio, M., Klysik, E., Johnson, R. L., et al. (2011). Hippo pathway inhibits Wnt signaling to restrain cardiomyocyte proliferation and heart size. Science 332, 458-461. doi: 10.1126/science.1199010

Hertig, V., Matos-Nieves, A., Garg, V., Villeneuve, L., Mamarbachi, M., Caland, L., et al. (2018). Nestin expression is dynamically regulated in cardiomyocytes during embryogenesis. J. Cell. Physiol. 233, 3218-3229. doi: 10.1002/jcp.26165

Hossain, Z., Ali, S. M., Ko, H. L., Xu, J., Ng, C. P., Guo, K., et al. (2007). Glomerulocystic kidney disease in mice with a targeted inactivation of Wwtr1. Proc. Natl. Acad. Sci. U.S.A. 104, 1631-1636. doi: 10.1073/pnas.0605266104

Hou, N., Wen, Y., Yuan, X., Xu, H., Wang, X., Li, F., et al. (2017). Activation of Yap1/Taz signaling in ischemic heart disease and dilated cardiomyopathy. Exp. Mol. Pathol. 103, 267-275. doi: 10.1016/j.yexmp.2017.11.006

Hsu, H.-T., Estaras, C., Huang, L., and Jones, K. A. (2018). Specifying the anterior primitive streak by modulating YAP1 levels in human pluripotent stem cells. Stem Cell Rep. 11, 1357-1364. doi: 10.1016/j.stemcr.2018.10.013

Hu, J., Li, Z., Xu, L. T., Sun, A. J., Fu, X. Y., Zhang, L., et al. (2015). Protective effect of apigenin on ischemia/reperfusion injury of the isolated rat heart. Cardiovasc. Toxicol. 15, 241-249. doi: 10.1007/s12012-014-9290-y

Ikeda, S., Mizushima, W., Sciarretta, S., Abdellatif, M., Zhai, P., Mukai, R., et al. (2019a). Hippo deficiency leads to cardiac dysfunction accompanied by cardiomyocyte dedifferentiation during pressure overload. Circ. Res. 124, 292-305. doi: 10.1161/CIRCRESAHA.118.314048

Ikeda, S., Mukai, R., Mizushima, W., Zhai, P., Oka, S. I., Nakamura, M., et al. (2019b). Yes-associated protein (YAP) facilitates pressure overload-induced dysfunction in the diabetic heart. JACC Basic Transl. Sci. 4, 611-622. doi: 10.1016/j.jacbts.2019.05.006

Jin, B., Zhu, J., Shi, H.-M., Wen, Z.-C., and Wu, B.-W. (2019). YAP activation promotes the transdifferentiation of cardiac fibroblasts to myofibroblasts in matrix remodeling of dilated cardiomyopathy. Br. J. Med. Biol. Res. 52:e7914. doi: 10.1590/1414-431X20187914

Kadoch, C., and Crabtree, G. R. (2015). Mammalian SWI/SNF chromatin remodeling complexes and cancer: mechanistic insights gained from human genomics. Sci. Adv. 1:e1500447. doi: 10.1126/sciadv.1500447

Khalafalla, F. G., Greene, S., Khan, H., Ilves, K., Monsanto, M. M., Alvarez, R. Jr., et al. (2017). P2Y(2) nucleotide receptor prompts human cardiac progenitor cell activation by modulating hippo signaling. Circ. Res. 121, 1224-1236. doi: 10.1161/circresaha.117.310812

Khan, K., Makhoul, G., Yu, B., Schwertani, A., and Cecere, R. (2019). The cytoprotective impact of yes-associated protein 1 after ischemia-reperfusion injury in AC16 human cardiomyocytes. Exp. Biol. Med. (Maywood) 244, 802812. doi: $10.1177 / 1535370219851243$

Khurana, S., Margamuljana, L., Joseph, C., Schouteden, S., Buckley, S. M., and Verfaillie, C. M. (2013). Glypican-3-mediated inhibition of CD26 by TFPI: a novel mechanism in hematopoietic stem cell homing and maintenance. Blood 121, 2587-2595. doi: 10.1182/blood-2012-09-456715

Kim, M., Kim, M., Park, S. J., Lee, C., and Lim, D. S. (2016). Role of angiomotinlike 2 mono-ubiquitination on YAP inhibition. EMBO Rep. 17, 64-78. doi: 10.15252/embr.201540809

Kim, M. K., Jang, J. W., and Bae, S. C. (2018). DNA binding partners of YAP/TAZ. BMB Rep. 51, 126-133. doi: 10.5483/bmbrep.2018.51.3.015

Kofler, M., Speight, P., Little, D., Di Ciano-Oliveira, C., Szaszi, K., and Kapus, A. (2018). Mediated nuclear import and export of TAZ and the underlying molecular requirements. Nat. Commun. 9:4966. doi: 10.1038/s41467-01807450-0

Kubin, T., Poling, J., Kostin, S., Gajawada, P., Hein, S., Rees, W., et al. (2011). Oncostatin $\mathrm{M}$ is a major mediator of cardiomyocyte dedifferentiation and remodeling. Cell Stem Cell 9, 420-432. doi: 10.1016/j.stem.2011.08.013

Kubli, D. A., Zhang, X., Lee, Y., Hanna, R. A., Quinsay, M. N., Nguyen, C. K., et al. (2013). Parkin protein deficiency exacerbates cardiac injury and reduces survival following myocardial infarction. J. Biol. Chem. 288, 915-926. doi: 10. 1074/jbc.M112.411363
Lai, J. K. H., Collins, M. M., Uribe, V., Jimenez-Amilburu, V., Guenther, S., Maischein, H.-M., et al. (2018). The Hippo pathway effector Wwtr1 regulates cardiac wall maturation in zebrafish. Development 145:dev159210. doi: 10.1242/ dev. 159210

Lange, A. W., Haitchi, H. M., Lecras, T. D., Sridharan, A., Xu, Y., Wert, S. E., et al. (2014). Sox17 is required for normal pulmonary vascular morphogenesis. Dev. Biol. 387, 109-120. doi: 10.1016/j.ydbio.2013.11.018

Leach, J. P., Heallen, T., Zhang, M., Rahmani, M., Morikawa, Y., Hill, M. C., et al. (2017). Hippo pathway deficiency reverses systolic heart failure after infarction. Nature 550, 260-264. doi: 10.1038/nature24045

Lee, J., Go, Y., Kang, I., Han, Y. M., and Kim, J. (2010). Oct-4 controls cell-cycle progression of embryonic stem cells. Biochem. J. 426, 171-181. doi: 10.1042/ BJ20091439

Levy, D., Adamovich, Y., Reuven, N., and Shaul, Y. (2008). Yapl phosphorylation by c-Abl is a critical step in selective activation of proapoptotic genes in response to DNA damage. Mol. Cell 29, 350-361. doi: 10.1016/j.molcel.2007.12.022

Li, F., Wang, X., Capasso, J. M., and Gerdes, A. M. (1996). Rapid transition of cardiac myocytes from hyperplasia to hypertrophy during postnatal development. J. Mol. Cell Cardiol. 28, 1737-1746. doi: 10.1006/jmcc.1996.0163

Li, L., Liu, T., Li, Y., Wu, C., Luo, K., Yin, Y., et al. (2018a). The deubiquitinase USP9X promotes tumor cell survival and confers chemoresistance through YAP1 stabilization. Oncogene 37, 2422-2431. doi: 10.1038/s41388-018-0134-2

Li, Y. W., Xu, J., Zhu, G. Y., Huang, Z. J., Lu, Y., Li, X. Q., et al. (2018b). Apigenin suppresses the stem cell-like properties of triple-negative breast cancer cells by inhibiting YAP/TAZ activity. Cell Death Discov. 4:105. doi: 10.1038/s41420018-0124-8

Li, Z., Zhao, B., Wang, P., Chen, F., Dong, Z., Yang, H., et al. (2010). Structural insights into the YAP and TEAD complex. Genes Dev. 24, 235-240. doi: 10. $1101 /$ gad.1865810

Liao, R., Jain, M., Cui, L., D’agostino, J., Aiello, F., Luptak, I., et al. (2002). Cardiacspecific overexpression of GLUT1 prevents the development of heart failure attributable to pressure overload in mice. Circulation 106, 2125-2131. doi: 10.1161/01.cir.0000034049.61181.f3

Lin, C., and Xu, X. (2017). YAP1-TEAD1-Glut1 axis dictates the oncogenic phenotypes of breast cancer cells by modulating glycolysis. Biomed. Pharmacother. 95, 789-794. doi: 10.1016/j.biopha.2017.08.091

Lin, Z., Von Gise, A., Zhou, P., Gu, F., Ma, Q., Jiang, J., et al. (2014). Cardiac-specific YAP activation improves cardiac function and survival in an experimental murine MI model. Circ. Res. 115, 354-363. doi: 10.1161/ CIRCRESAHA.115.303632

Lin, Z., Zhou, P., Von Gise, A., Gu, F., Ma, Q., Chen, J., et al. (2015). Pi3kcb links Hippo-YAP and PI3K-AKT signaling pathways to promote cardiomyocyte proliferation and survival. Circ. Res. 116, 35-45. doi: 10.1161/CIRCRESAHA. 115.304457

Liu, A., Zeng, S., Lu, X., Xiong, Q., Xue, Y., Tong, L., et al. (2019). Overexpression of G2 and S phase-expressed-1 contributes to cell proliferation, migration, and invasion via regulating p53/FoxM1/CCNB1 pathway and predicts poor prognosis in bladder cancer. Int. J. Biol. Macromol. 123, 322-334. doi: 10.1016/ j.ijbiomac.2018.11.032

Liu, C. Y., Lv, X., Li, T., Xu, Y., Zhou, X., Zhao, S., et al. (2011). PP1 cooperates with ASPP2 to dephosphorylate and activate TAZ. J. Biol. Chem. 286, 5558-5566. doi: 10.1074/jbc.M110.194019

Liu, S., Bou, G., Zhao, J., Guo, S., Guo, J., Weng, X., et al. (2018). Asynchronous CDX2 expression and polarization of porcine trophoblast cells reflects a speciesspecific trophoderm lineage determination progress model. Mol. Reprod. Dev. 85, 590-598. doi: 10.1002/mrd.22994

Liu-Chittenden, Y., Huang, B., Shim, J. S., Chen, Q., Lee, S. J., Anders, R. A., et al. (2012). Genetic and pharmacological disruption of the TEAD-YAP complex suppresses the oncogenic activity of YAP. Genes Dev. 26, 1300-1305. doi: 10. 1101/gad.192856.112

Ma, S., and Dong, Z. (2019). Melatonin attenuates cardiac reperfusion stress by improving OPA1-related mitochondrial fusion in a Yap-Hippo pathwaydependent manner. J. Cardiovasc. Pharmacol. 73, 27-39. doi: 10.1097/FJC. 0000000000000626

Maejima, Y., Kyoi, S., Zhai, P., Liu, T., Li, H., Ivessa, A., et al. (2013). Mst1 inhibits autophagy by promoting the interaction between Beclin1 and Bcl-2. Nat. Med. 19, 1478-1488. doi: $10.1038 / \mathrm{nm} .3322$ 
Makita, R., Uchijima, Y., Nishiyama, K., Amano, T., Chen, Q., Takeuchi, T., et al. (2008). Multiple renal cysts, urinary concentration defects, and pulmonary emphysematous changes in mice lacking TAZ. Am. J. Physiol. Renal. Physiol. 294, F542-F553. doi: 10.1152/ajprenal.00201.2007

Matsuda, T., Zhai, P., Sciarretta, S., Zhang, Y., Jeong, J. I., Ikeda, S., et al. (2016). NF2 activates Hippo signaling and promotes ischemia/reperfusion injury in the heart. Circ. Res. 119, 596-606. doi: 10.1161/CIRCRESAHA.116.30 8586

Matsui, Y., Nakano, N., Shao, D., Gao, S., Luo, W., Hong, C., et al. (2008). Lats2 is a negative regulator of myocyte size in the heart. Circ. Res. 103, 1309-1318. doi: 10.1161/CIRCRESAHA.108.180042

Meng, Z., Moroishi, T., and Guan, K. L. (2016). Mechanisms of Hippo pathway regulation. Genes Dev. 30, 1-17. doi: 10.1101/gad.274027.115

Miskolczi, Z., Smith, M. P., Rowling, E. J., Ferguson, J., Barriuso, J., and Wellbrock, C. (2018). Collagen abundance controls melanoma phenotypes through lineage-specific microenvironment sensing. Oncogene 37, 3166-3182. doi: 10.1038/s41388-018-0209-0

Mo, J.-S. (2017). The role of extracellular biophysical cues in modulating the Hippo-YAP pathway. BMB Rep. 50, 71-78. doi: 10.5483/bmbrep.2017.50. 2.199

Monroe, T. O., Hill, M. C., Morikawa, Y., Leach, J. P., Heallen, T., Cao, S., et al. (2019). YAP partially reprograms chromatin accessibility to directly induce adult cardiogenesis in vivo. Dev. Cell 48, 765-779.e7. doi: 10.1016/j.devcel.2019. 01.017

Moon, S., Kim, W., Kim, S., Kim, Y., Song, Y., Bilousov, O., et al. (2017). Phosphorylation by NLK inhibits YAP-14-3-3-interactions and induces its nuclear localization. EMBO Rep. 18, 61-71. doi: 10.15252/embr.201642683

Morikawa, Y., Heallen, T., Each, J. L., Xiao, Y., and Martin, J. F. (2017). Dystrophinglycoprotein complex sequesters Yap to inhibit cardiomyocyte proliferation. Nature 547, 227-231. doi: 10.1038/nature22979

Moroishi, T., Park, H. W., Qin, B., Chen, Q., Meng, Z., Plouffe, S. W., et al. (2015). A YAP/TAZ-induced feedback mechanism regulates Hippo pathway homeostasis. Genes Dev. 29, 1271-1284. doi: 10.1101/gad.262816.115

Moya, I. M., and Halder, G. (2018). Hippo-YAP/TAZ signalling in organ regeneration and regenerative medicine. Nat. Rev. Mol. Cell Biol. 20, 211-226. doi: 10.1038/s41580-018-0086-y

Nakamura, M., Zhai, P., Del Re, D. P., Maejima, Y., and Sadoshima, J. (2016). Mst1mediated phosphorylation of $\mathrm{Bcl}-\mathrm{xL}$ is required for myocardial reperfusion injury. JCI Insight 1:e86217.

Nasrollahi, S., and Pathak, A. (2017). Hydrogel-based microchannels to measure confinement- and stiffness-sensitive Yes-associated-protein activity in epithelial clusters. Mrs Commun. 7, 450-457. doi: 10.1557/mrc.2017.87

Negron-Perez, V. M., and Hansen, P. J. (2018). Role of yes-associated protein 1, angiomotin, and mitogen-activated kinase kinase $1 / 2$ in development of the bovine blastocyst. Biol. Reprod. 98, 170-183. doi: 10.1093/biolre/iox172

Nishida, K., Yamaguchi, O., and Otsu, K. (2008). Crosstalk between autophagy and apoptosis in heart disease. Circ. Res. 103, 343-351. doi: 10.1161/CIRCRESAHA. 108.175448

Odashima, M., Usui, S., Takagi, H., Hong, C., Liu, J., Yokota, M., et al. (2007). Inhibition of endogenous Mst1 prevents apoptosis and cardiac dysfunction without affecting cardiac hypertrophy after myocardial infarction. Circ. Res. 100, 1344-1352. doi: 10.1161/01.res.0000265846.23485.7a

Oudhoff, M. J., Freeman, S. A., Couzens, A. L., Antignano, F., Kuznetsova, E., Min, P. H., et al. (2013). Control of the Hippo pathway by Set7-dependent methylation of Yap. Dev. Cell 26, 188-194. doi: 10.1016/j.devcel.2013.0 5.025

Pan, D. (2007). Hippo signaling in organ size control. Genes Dev. 21, 886-897. doi: 10.1101/gad.1536007

Paramasivam, M., Sarkeshik, A., Yates, J. R. III, Fernandes, M. J., and Mccollum, D. (2011). Angiomotin family proteins are novel activators of the LATS2 kinase tumor suppressor. Mol. Biol. Cell 22, 3725-3733. doi: 10.1091/mbc.E11-040300

Peng, C., Zhu, Y., Zhang, W., Liao, Q., Chen, Y., Zhao, X., et al. (2017). Regulation of the Hippo-YAP pathway by glucose sensor O-GlcNAcylation. Mol. Cell 68:e595. doi: 10.1016/j.molcel.2017.10.010

Petroski, M. D., and Deshaies, R. J. (2005). Function and regulation of cullin-RING ubiquitin ligases. Nat. Rev. Mol. Cell Biol. 6, 9-20. doi: 10.1038/nrm1547
Piccolo, S., Dupont, S., and Cordenonsi, M. (2014). The biology of Yap/Taz: Hippo signaling and beyond. Physiol. Rev. 94, 1287-1312. doi: 10.1152/physrev.00005. 2014

Ponnusamy, M., Li, P.-F., and Wang, K. (2017). Understanding cardiomyocyte proliferation: an insight into cell cycle activity. Cell. Mol. Life Sci. 74, 1019-1034. doi: 10.1007/s00018-016-2375-y

Qin, Z., Xia, W., Fisher, G. J., Voorhees, J. J., and Quan, T. (2018). YAP/TAZ regulates TGF-beta/Smad3 signaling by induction of Smad7 via AP-1 in human skin dermal fibroblasts. Cell Commun. Signal. 16:18. doi: 10.1186/s12964-0180232-3

Ragni, C. V., Diguet, N., Le Garrec, J.-F., Novotova, M., Resende, T. P., Pop, S., et al. (2017). Amotll mediates sequestration of the Hippo effector Yap1 downstream of Fat4 to restrict heart growth. Nat. Commun. 8:14582. doi: 10. $1038 /$ ncomms 14582

Roos, W. P., and Kaina, B. (2013). DNA damage-induced cell death: from specific DNA lesions to the DNA damage response and apoptosis. Cancer Lett. 332, 237-248. doi: 10.1016/j.canlet.2012.01.007

Rosenbluh, J., Nijhawan, D., Cox, A. G., Li, X., Neal, J. T., Schafer, E. J., et al. (2012). beta-Catenin-driven cancers require a YAP1 transcriptional complex for survival and tumorigenesis. Cell 151, 1457-1473. doi: 10.1016/j.cell.2012.11.026

Schindler, Y. L., Garske, K. M., Wang, J., Firulli, B. A., Firulli, A. B., Poss, K. D., et al. (2014). Hand2 elevates cardiomyocyte production during zebrafish heart development and regeneration. Development 141, 3112-3122. doi: 10.1242/dev. 106336

Shao, D., Zhai, P., Del Re, D. P., Sciarretta, S., Yabuta, N., Nojima, H., et al. (2014). A functional interaction between Hippo-YAP signalling and FoxO1 mediates the oxidative stress response. Nat. Commun. 5:3315. doi: 10.1038/ncomms4315

Srivastava, D., and Yu, S. (2006). Stretching to meet needs: integrin-linked kinase and the cardiac pump. Genes Dev. 20, 2327-2331. doi: 10.1101/gad.1472506

Stelitano, D., Peche, L. Y., Dalla, E., Monte, M., Piazza, S., and Schneider, C. (2017). GTSE1: a novel TEAD4-E2F1 target gene involved in cell protrusions formation in triple-negative breast cancer cell models. Oncotarget 8, 67422-67438. doi: 10.18632/oncotarget.18691

Sudol, M. (1994). Yes-associated protein (YAP65) is a proline-rich phosphoprotein that binds to the SH3 domain of the Yes proto-oncogene product. Oncogene 9, 2145-2152.

Tam, P. P., Parameswaran, M., Kinder, S. J., and Weinberger, R. P. (1997). The allocation of epiblast cells to the embryonic heart and other mesodermal lineages: the role of ingression and tissue movement during gastrulation. Development 124, 1631-1642.

Tamm, C., Bower, N., and Anneren, C. (2011). Regulation of mouse embryonic stem cell self-renewal by a Yes-YAP-TEAD2 signaling pathway downstream of LIF. J. Cell Sci. 124, 1136-1144. doi: 10.1242/jcs.075796

Tang, C., Takahashi-Kanemitsu, A., Kikuchi, I., Ben, C., and Hatakeyama, M. (2018). Transcriptional co-activator functions of YAP andTAZ are inversely regulated by tyrosine phosphorylation status of parafibromin. iScience 1, 1-15. doi: 10.1016/j.isci.2018.01.003

Tang, Z., Ma, Q., Wang, L., Liu, C., Gao, H., Yang, Z., et al. (2019). A brief review: some compounds targeting YAP against malignancies. Future Oncol. 15, 1535-1543. doi: 10.2217/fon-2019-0035

Taniguchi, K., Wu, L. W., Grivennikov, S. I., De Jong, P. R., Lian, I., Yu, F. X., et al. (2015). A gp130-Src-YAP module links inflammation to epithelial regeneration. Nature 519, 57-62. doi: 10.1038/nature14228

Tao, G., Kahr, P. C., Morikawa, Y., Zhang, M., Rahmani, M., Heallen, T. R., et al. (2016). Pitx2 promotes heart repair by activating the antioxidant response after cardiac injury. Nature 534, 119-123. doi: 10.1038/nature17959

Tian, Y., Liu, Y., Wang, T., Zhou, N., Kong, J., Chen, L., et al. (2015). A microRNA-Hippo pathway that promotes cardiomyocyte proliferation and cardiac regeneration in mice. Sci. Transl. Med. 7:279ra38. doi: 10.1126/ scitranslmed.3010841

Tu, K., Yang, W., Li, C., Zheng, X., Lu, Z., Guo, C., et al. (2014). Fbxw7 is an independent prognostic marker and induces apoptosis and growth arrest by regulating YAP abundance in hepatocellular carcinoma. Mol. Cancer 13:110. doi: 10.1186/1476-4598-13-110

Valis, K., Talacko, P., Grobarova, V., Cerny, J., and Novak, P. (2016). Shikonin regulates C-MYC and GLUT1 expression through the MST1-YAP1-TEAD1 axis. Exp. Cell Res. 349, 273-281. doi: 10.1016/j.yexcr.2016.10.018 
Vassort, G. (2001). Adenosine 5'-triphosphate: a P2-purinergic agonist in the myocardium. Physiol. Rev. 81, 767-806. doi: 10.1152/physrev.2001.81.2.767

von Gise, A., Lin, Z., Schlegelmilch, K., Honor, L. B., Pan, G. M., Buck, J. N., et al. (2012). YAP1, the nuclear target of Hippo signaling, stimulates heart growth through cardiomyocyte proliferation but not hypertrophy. Proc. Natl. Acad. Sci. U.S.A. 109, 2394-2399. doi: 10.1073/pnas.1116136109

Wang, J., Ye, C., Chen, C., Xiong, H., Xie, B., Zhou, J., et al. (2017). Glucose transporter GLUT1 expression and clinical outcome in solid tumors: a systematic review and meta-analysis. Oncotarget 8, 16875-16886. doi: 10.18632/ oncotarget.15171

Wang, P., Bai, Y., Song, B., Wang, Y., Liu, D., Lai, Y., et al. (2011). PP1A-mediated dephosphorylation positively regulates YAP2 activity. PLoS One 6:e24288. doi: 10.1371/journal.pone.0024288

Wang, X., Ha, T., Liu, L., Hu, Y., Kao, R., Kalbfleisch, J., et al. (2018). TLR3 mediates repair and regeneration of damaged neonatal heart through glycolysis dependent YAP1 regulated miR-152 expression. Cell Death Differ. 25, 966-982. doi: 10.1038/s41418-017-0036-9

Wang, Y., Hu, G., Liu, F., Wang, X., Wu, M., Schwarz, J. J., et al. (2014). Deletion of yes-associated protein (YAP) specifically in cardiac and vascular smooth muscle cells reveals a crucial role for YAP in mouse cardiovascular development. Circ. Res. 114, 957-965. doi: 10.1161/CIRCRESAHA.114.303411

Xiao, Y., Hill, M. C., Zhang, M., Martin, T. J., Morikawa, Y., Wang, S., et al. (2018). Hippo signaling plays an essential role in cell state transitions during cardiac fibroblast development. Dev. Cell 45, 153-169.e6. doi: 10.1016/j.devcel.2018.0 3.019

Xin, M., Kim, Y., Sutherland, L. B., Murakami, M., Qi, X., Mcanally, J., et al. (2013a). Hippo pathway effector Yap promotes cardiac regeneration. Proc. Natl. Acad. Sci. U.S.A. 110, 13839-13844. doi: 10.1073/pnas.1313192110

Xin, M., Kim, Y., Sutherland, L. B., Qi, X., Mcanally, J., Schwartz, R. J., et al. (2011). Regulation of insulin-like growth factor signaling by Yap governs cardiomyocyte proliferation and embryonic heart size. Sci. Signal. 4:ra70. doi: $10.1126 /$ scisignal.2002278

Xin, M., Olson, E. N., and Bassel-Duby, R. (2013b). Mending broken hearts: cardiac development as a basis for adult heart regeneration and repair. Nat. Rev. Mol. Cell Biol. 14, 529-541. doi: 10.1038/nrm3619

Yamamoto, S., Yang, G., Zablocki, D., Liu, J., Hong, C., Kim, S. J., et al. (2003). Activation of Mst1 causes dilated cardiomyopathy by stimulating apoptosis without compensatory ventricular myocyte hypertrophy. J. Clin. Invest. 111, 1463-1474. doi: 10.1172/jci17459

Yang, Y., Del Re, D. P., Nakano, N., Sciarretta, S., Zhai, P., Park, J., et al. (2015). miR-206 mediates YAP-induced cardiac hypertrophy and survival. Circ. Res. 117, 891-904. doi: 10.1161/CIRCRESAHA.115.306624

Yao, F., Zhou, Z., Kim, J., Hang, Q., Xiao, Z., Ton, B. N., et al. (2018). SKP2and OTUD1-regulated non-proteolytic ubiquitination of YAP promotes YAP nuclear localization and activity. Nat. Commun. 9:2269. doi: 10.1038/s41467018-04620-y

Zhang, Q., Han, X., Chen, J., Xie, X., Xu, J., Zhao, Y., et al. (2018a). Yes-associated protein (YAP) and transcriptional coactivator with PDZ-binding motif (TAZ) mediate cell density-dependent proinflammatory responses. J. Biol. Chem. 293, 18071-18085. doi: 10.1074/jbc.RA118.004251

Zhang, Q., Zhang, Y., Parsels, J. D., Lohse, I., Lawrence, T. S., Pasca Di Magliano, M., et al. (2016). Fbxw7 deletion accelerates kras(G12D)-driven pancreatic tumorigenesis via Yap accumulation. Neoplasia 18, 666-673. doi: 10.1016/j.neo. 2016.08.009

Zhang, X., Li, Y., Ma, Y., Yang, L., Wang, T., Meng, X., et al. (2018b). Yes-associated protein (YAP) binds to HIF-1 alpha and sustains HIF-1 alpha protein stability to promote hepatocellular carcinoma cell glycolysis under hypoxic stress. J. Exp. Clin. Cancer Res. 37:216. doi: 10.1186/s13046-018-0892-2

Zhang, X., Qiao, Y., Wu, Q., Chen, Y., Zou, S., Liu, X., et al. (2017). The essential role of YAP O-GlcNAcylation in high-glucose-stimulated liver tumorigenesis. Nat. Commun. 8:15280. doi: 10.1038/ncomms 15280

Zhao, B., Li, L., Tumaneng, K., Wang, C. Y., and Guan, K. L. (2010). A coordinated phosphorylation by Lats and CK1 regulates YAP stability through SCF(betaTRCP). Genes Dev. 24, 72-85. doi: 10.1101/gad.1843810

Zhao, B., Wei, X., Li, W., Udan, R. S., Yang, Q., Kim, J., et al. (2007). Inactivation of YAP oncoprotein by the Hippo pathway is involved in cell contact inhibition and tissue growth control. Genes Dev. 21, 2747-2761. doi: 10.1101/gad.1602907

Zhao, W., Liu, H., Wang, J., Wang, M., and Shao, R. (2018). Cyclizing-berberine A35 induces G2/M arrest and apoptosis by activating YAP phosphorylation (Ser127). J. Exp. Clin. Cancer Res. 37:98. doi: 10.1186/s13046-018-0759-6

Zhao, W. B., Lu, Q., Nguyen, M. N., Su, Y., Ziemann, M., Wang, L. N., et al. (2019). Stimulation of beta-adrenoceptors up-regulates cardiac expression of galectin-3 and BIM through the Hippo signalling pathway. Br. J. Pharmacol. 176, 2465-2481. doi: 10.1111/bph.14674

Zhou, Q., Li, L., Zhao, B., and Guan, K. L. (2015). The hippo pathway in heart development, regeneration, and diseases. Circ. Res. 116, 1431-1447. doi: 10. 1161/circresaha.116.303311

Zhou, Z., Zhang, Y., Lin, L., and Zhou, J. (2018). Apigenin suppresses the apoptosis of $\mathrm{H} 9 \mathrm{C} 2$ rat cardiomyocytes subjected to myocardial ischemiareperfusion injury via upregulation of the PI3K/Akt pathway. Mol. Med. Rep. 18, 1560-1570. doi: 10.3892/mmr.2018.9115

Zou, J., Ma, W., Li, J., Littlejohn, R., Zhou, H., Kim, I.-M., et al. (2018). Neddylation mediates ventricular chamber maturation through repression of Hippo signaling. Proc. Natl. Acad. Sci. U.S.A. 115, E4101-E4110. doi: 10.1073/ pnas. 1719309115

Conflict of Interest: The authors declare that the research was conducted in the absence of any commercial or financial relationships that could be construed as a potential conflict of interest.

Copyright (c) 2020 Chen, Li, Luo and Hou. This is an open-access article distributed under the terms of the Creative Commons Attribution License (CC BY). The use, distribution or reproduction in other forums is permitted, provided the original author(s) and the copyright owner(s) are credited and that the original publication in this journal is cited, in accordance with accepted academic practice. No use, distribution or reproduction is permitted which does not comply with these terms. 